\title{
Cellular Physiology

\section{Gender Difference in Proteome of Brown Adipose Tissues between Male and Female Rats Exposed to a High Fat Diet}

\author{
Duk Kwon Choi, Tae Seok Oh, Jung-Won Choi, Rajib Mukherjee, \\ Xia Wang, Hao Liu and Jong Won Yun
}

Department of Biotechnology, Daegu University, Kyungsan, Kyungbuk

\section{Key Words}

2-DE - Gender differences $\cdot$ High fat diet - Obesity • Proteome $\cdot$ Brown adipose tissue

\begin{abstract}
Background: Although the importance of gender as a key determinant in health and illness has been recognized for a long time, systematic studies of gender differences in medicine are still lacking. We hypothesized that interscapular brown adipocyte tissue (BAT), is not only a key tissue contributing to energy expenditure, but also regulates diet-induced thermogenesis, and may be an ideal target for studying gender differences in obesity development in response to a high fat diet (HFD). Methods: We therefore performed differential proteome analysis of BAT from lean and obese rats of both genders fed a HFD using 2-DE combined with MALDI-TOF-MS. Results: When exposed to a HFD, male rats gained more body weight with increased values of plasma biochemical parameters than did female rats. Among 595 matched spots, 48 differentially expressed identified spots showed significant gender differences, whereas 7 proteins showed no gender differences, but did show a HFD response. Conclusions: Proteomic
\end{abstract}

\section{KARGER}

Fax +4161306 1234

E-Mail karger@karger.ch

www.karger.com
(C) 2011 S. Karger AG, Basel

$1015-8987 / 11 / 0285-0933 \$ 38.00 / 0$

Accessible online at:

www.karger.com/cpb investigations into gender-dimorphic protein modulation in BAT may provide conclusive results showing higher expression of numerous proteins involved in thermogenesis and fat oxidation as well as lower expression of proteins contributing to fat synthesis in female rats than in male rats.

Copyright @ 2011 S. Karger AG, Basel

\section{Introduction}

A broader knowledge of gender differences would provide a basis for specific evidence-based interventions, allowing for prevention and treatment of many diseases by matching the different needs of women and men, and contribute to the development of healthcare policy [1]. Although many factors, including social and cultural environment, are considered to contribute to differences in obesity between women and men, biological differences are more profound [2].

To date, numerous studies have been conducted in efforts to address sex differences in relation to obesity, including the differences in body fat distribution [3-10], physical activity [1], metabolic rate [11-14], oxidative capacity [15], sex hormones [16-19], energy expenditure

Dr. Jong Won Yun

Department of Biotechnology, Daegu University

Kyungsan, Kyungbuk 712-714 (Republic of Korea)

Tel. +82-53-850-6556, Fax +82-53-850-6559

E-Mailjwyun@daegu.ac.kr 
[20-24], adipokine signaling [25-27], and appetite regulation [24, 28-30]. However, the role played by gender in susceptibility to obesity is not fully understood. Some evidence suggests that males have a higher susceptibility to becoming obese, compared with females [31-33]. However, some contradictory results have been found in the literature, which may be explained by differences between strains and gender, as well as differences in the nutritional state of the animals [1, 15, 18, 34-36].

Some evidence suggests that energy expenditure of females is greater than that of males due to higher thermogenic activation [22], greater oxidative capacity with more effective antioxidant machinery [34], and greater respiratory and phosphorylative capacities in mitochondria $[18,35,36]$. In this regard, BAT, as a key tissue contributing to energy expenditure, may be an ideal target for investigating gender differences in obesity development in response to a HFD.

To date, several proteomics studies have been conducted in an effort to reveal gender differences in protein expression patterns in blood [37, 38], muscle [39], kidney [40], brain [41], and sperm [42]. However, to the best of our knowledge, no comparative proteomic study in biofluids or metabolic organ tissues of lean and obese animal models for examination of protein abundance and/ or regulation patterns between genders has been conducted. Despite its important function in diet-induced thermogenesis, with the exception of two recently published results $[43,44]$, no studies of the BAT proteome with respect to obesity have not been conducted.

Therefore, in the present study, we performed differential proteome analysis of interscapular BAT to determine sex-specific susceptibility to obesity in male and female rats, and to identify proteins determining phenotypic differences between genders.

\section{Materials and Methods}

\section{Animals and breeding conditions}

Male and female SLC Sprague-Dawley (SD) rats (5 weeks of age) were purchased from Daehan Experimental Animals (Seoul, Korea) and housed one per cage in a temperature $(23 \pm$ $2{ }^{\circ} \mathrm{C}$ ) and humidity (55\%)-controlled room with a $12 \mathrm{hr}$ light/ dark cycle. Rats were provided free access to standard chow and tap water for an adaptation period of 1 week. Male and female rats were randomly divided into 2 groups, with 20 rats fed a ND ( $12 \%$ calories from fat; control group) and 40 rats fed a HFD ( $45 \%$ calories from fat), respectively. Rat feeds were purchased from Feed Korea Lab (Hanam, Korea); the dietary compositions of these feeds are shown in Table 1. All rats and feeds were weighed every week for 8 weeks. Rats were food

\begin{tabular}{|c|c|c|}
\hline Ingredient & ND & HFD \\
\hline & \multicolumn{2}{|c|}{ Composition by weight; $\mathrm{g} / \mathrm{kg}$} \\
\hline Casein & 200 & 200 \\
\hline Cornstarch & 150 & 155 \\
\hline Sucrose & 500 & 50 \\
\hline Dextrose & 0 & 132 \\
\hline Cellulose & 50 & 50 \\
\hline Soybean oil & 0 & 25 \\
\hline Corn oil & 50 & 0 \\
\hline Lard & 0 & 175 \\
\hline Mineral mix & 35 & 35 \\
\hline Vitamin mix ${ }^{b}$ & 10 & 10 \\
\hline $\mathrm{TBHQ}^{\mathrm{c})}$ & 0 & 0.014 \\
\hline DL-Methionin & 3 & 0 \\
\hline L-cystine & 0 & 3 \\
\hline \multirow[t]{2}{*}{ Choline bitartrate } & 2 & 2.5 \\
\hline & \multicolumn{2}{|c|}{ Composition by calories; $\%$} \\
\hline Protein & 21 & 20 \\
\hline Carbohydrate & 68 & 35 \\
\hline Fat & 12 & 45 \\
\hline Total & $3902 \mathrm{kcal} / \mathrm{kg}$ & $4776 \mathrm{kcal} / \mathrm{kg}$ \\
\hline
\end{tabular}

Table 1. Composition of diets used in this study. ${ }^{\text {a) Mineral }}$ mix; sucrose (118.03 g/kg), calcium phosphate dibasic (500 g/ $\mathrm{kg})$, sodium chloride $(74 \mathrm{~g} / \mathrm{kg})$, potassium citrate $(220 \mathrm{~g} / \mathrm{kg})$, potassium sulfate $(52 \mathrm{~g} / \mathrm{kg})$, magnesium oxide $(24 \mathrm{~g} / \mathrm{kg})$, magnesium oxide $(24 \mathrm{~g} / \mathrm{kg})$, manganous carbonate $(3.5 \mathrm{~g} / \mathrm{kg})$, ferric citrate $(6 \mathrm{~g} / \mathrm{kg})$, zinc carbonate $(1.6 \mathrm{~g} / \mathrm{kg})$, cupric carbonate $(0.3 \mathrm{~g} / \mathrm{kg})$, potassium iodate $(0.01 \mathrm{~g} / \mathrm{kg})$, sodium selenite $(0.01$

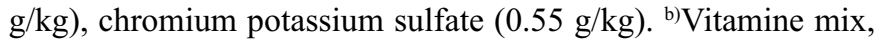
sucrose $(981.15 \mathrm{~g} / \mathrm{kg})$, thiamine $(0.6 \mathrm{~g} / \mathrm{kg})$, vitamin E acetate $(500 \mathrm{IU} / \mathrm{g} ; 10 \mathrm{~g} / \mathrm{kg})$, niacin $(3 \mathrm{~g} / \mathrm{kg})$, calcium pantothenate $(1.6$ $\mathrm{g} / \mathrm{kg})$, vitamin B12 $(0.1 \% ; 1 \mathrm{~g} / \mathrm{kg})$, vitamin A palmitate $(500,000$ $\mathrm{IU} / \mathrm{g} ; 0.8 \mathrm{~g} / \mathrm{kg})$, pyridoxine $(0.7 \mathrm{~g} / \mathrm{kg})$, riboflavin $(0.6 \mathrm{~g} / \mathrm{kg})$, vitamin D3 (400,000 IU/g; $0.25 \mathrm{~g} / \mathrm{kg})$, folic acid $(0.2 \mathrm{~g} / \mathrm{kg})$, menadione sodium bisulfate $(0.08 \mathrm{~g} / \mathrm{kg})$, biotin $(0.02 \mathrm{~g} / \mathrm{kg})$. ${ }^{c}$ TBHQ, tert-butylhydroquinone.

deprived for at least $12 \mathrm{~h}$ before sacrifice and were anesthetized using 3\% diethyl ether. These experiments were approved by the Committee for Laboratory Animal Care and Use of Daegu University. All procedures were conducted in accordance with the Guide for the Care and Use of Laboratory Animals published by the National Institutes of Health.

\section{Blood plasma samples}

Blood samples were obtained by resection at the end of the tail of the rat under anesthesia and collected into EDTAtubes (BD, Franklin Lakes, NJ, USA). Plasma was separated by centrifugation $(3,000 \times g, 10 \mathrm{~min})$, followed by storage at $-80^{\circ} \mathrm{C}$ 
until further analysis. Protein concentration of plasma was determined by the Bradford method [45] using protein assay dye reagent concentrate (Bio-Rad, Hercules, CA, USA).

\section{Plasma biochemical parameters}

Plasma biochemical parameters were measured using plasma of ND-fed rats $(n=7)$ and HFD-fed rats $(n=7)$ from male and female rats, respectively. Commercial kits purchased from Bio Clinical System Corporation (Anyang, Korea) were used for enzymatic measurement of total cholesterol and high density lipoprotein (HDL) cholesterol in plasma, and enzymatic kits from Asan Pharmaceutical (Seoul, Korea) were used for determination of triglycerides (TGs) and glucose. An enzymatic fatty acid quantification kit (Abcam, Cambridge, UK) was used for quantification of free fatty acids (FFA). A Rat Insulin kit (Millipore Co., Billerica, MA, USA) was used for measurement of plasma insulin levels, and leptin levels were determined by use of the Rat Leptin kit (Millipore Co., Billerica, MA, USA), which is a sandwich enzyme-linked immunosorbent assay (ELISA) system. Sex hormone levels were measured using a Rat Estrogen ELISA kit (Cusabio Biotech. Co., LTD, Wuhan, Hubei, China) and a Rat Testosterone ELISA kit (Cusabio Biotech). Measurements were performed according to the manufacturer's instructions.

\section{Preparation of BAT samples for 2-DE}

Interscapular BAT of rats in each group was excised immediately after anesthetization of rats with diethyl ether after overnight fasting. The resulting tissues were then washed with a cold saline solution. BAT was pulverized under liquid nitrogen and stored at $-80^{\circ} \mathrm{C}$. Tissues were lysed in $200 \mu \mathrm{L}$ rehydration buffer solution containing $7 \mathrm{M}$ urea, $2 \mathrm{M}$ thiourea, 4\% CHAPS, 20 mM DTT, 1 mM PMSF, 2\% IPG buffer (Ampholyte 3-10, BioRad), and a trace of bromophenol blue. A homogenizer (PT 1200 E, Kinematica Ltd., Luzern, Switzerland) was used on ice. Extracts were centrifuged at $13,000 \times g$ for $20 \mathrm{~min}$ and the supernatant was then stored at $-80^{\circ} \mathrm{C}$ until analysis. Protein content of adipose tissues was determined by the Bradford method ${ }^{45}$ using Bradford reagent (Sigma-Aldrich, St. Louis, MO, USA).

\section{2-DE analysis}

2-DE was performed 3 times using pooled BAT protein samples from 7 rats per group, which consisted of the control group, which was fed an ND, and the obese group, which was fed an HFD for both male and female rats. The 2-DE experiments were conducted using previously described methods [44]. Briefly, immobilized $\mathrm{pH}$ gradient (IPG)-isoelectric focusing (IEF) of BAT samples was performed on $\mathrm{pH} 3-10$ and $18 \mathrm{~cm}$ IPG DryStrips (GE Healthcare, Buckinghamshire, UK) in a PROTEAN IEF Cell (Bio-Rad) using the protocol recommended by the manufacturer. IPG strips were passively rehydrated for $24 \mathrm{~h}$ in strip holders with $350 \mu \mathrm{L}$ of rehydration solution, which included $7 \mathrm{M}$ urea (Bio Basic, Ontario, Canada), $2 \mathrm{M}$ thiourea (Sigma-Aldrich), 4\% CHAPS (Bio Basic), 1 mM PMSF (SigmaAldrich), $20 \mathrm{mM}$ DTT (GE Healthcare), 2\% IPG buffer (Bio$\mathrm{Rad}$ ), and $150 \mu \mathrm{g}$ BAT protein. IEF was executed for $14 \mathrm{~h}$ as follows: $15 \mathrm{~min}$ at $250 \mathrm{~V}$ (Conditioning step), $3 \mathrm{~h}$ at 250-10,000 V

Gender Differences in Brown Adipose Tissues of Rats
(Voltage ramping), $6 \mathrm{~h}$ at $10,000 \mathrm{~V}$ (Final focusing), and then held at $5 \mathrm{~h} 500 \mathrm{~V}$ (Hold step) until running the second dimension. After focusing, gel strips were equilibrated twice in a solution containing $6 \mathrm{M}$ urea, 2\% SDS (Generay Biotech, Shanghai, China), 1\% DTT, 30\% glycerol (Bio Basic), and $50 \mathrm{mM}$ Tris- $\mathrm{HCl}$ (pH 6.8) for $20 \mathrm{~min}$. In the second equilibration buffer, DTT was replaced with $2.5 \%$ iodoacetamide (Bio-Rad) to remove excess DTT which causes point streaking in silver stained gels. Gel strips were then placed onto a $20 \times 20 \mathrm{~cm} 12 \%$ polyacrylamide gel for resolution in the second dimension. Fractionation was performed using the Laemmli SDS-discontinuous system at a constant voltage of $15 \mathrm{~mA}$ per gel for $14 \mathrm{~h}$. For image analysis and peptide mass fingerprinting (PMF), a total of 12 gels, including 3 gels per group with separated proteins were visualized by silver staining. Silver staining was performed as follows. Gels were fixed for $30 \mathrm{~min}$ in 50\% ethanol (DUKSAN Pure Chemicals, Ansan, Korea) and 5\% acetic acid (DUKSAN Pure Chemicals), followed by $10 \mathrm{~min}$ in $30 \%$ ethanol, and waterwashed 3 times, for $5 \mathrm{~min}$ per wash Gels were sensitized for 10 min in $0.02 \%$ sodium thiosulfate (Sigma-Aldrich), followed by 0.5 min water washes (3 times), and incubated for $25 \mathrm{~min}$ in $0.3 \%$ silver nitrate (Kojima Chemicals, Sayama, Japan). After two 0.5 min water washes, proteins were visualized with developing solution (3\% sodium carbonate; DUKSAN Pure Chemicals, Ansan, Korea), 0.02\% sodium thiosulfate, $0.05 \%$ formalin (DC Chemicals, Incheon, Korea), and stopped with $6 \%$ acetic acid.

\section{Image acquisition and data analysis}

Gels were imaged on a UMAX PowerLook 1120 (Maxium Technologies, Akron, OH, USA) and images were compared using modified ImageMaster 2-D software V4.95 (GE Healthcare). A reference gel was selected from gels of the normal group and spots detected from the other gels were matched with those in the reference gel. Relative optical density and relative volume were also calculated for the purpose of correction for differences in gel staining. Each spot intensity volume was processed by background subtraction and total spot volume normalization; the resulting spot volume percentage was used for comparison.

\section{Peptide mass fingerprinting (PMF)}

Spots were manually excised from the gels with autoclaved materials in a clean environment. Selected spots were digested with trypsin (Promega, Madison, WI, USA), mixed with $\alpha$-cyano-4-hydroxycinnamic acid in 50\% acetonitrile/ $0.1 \%$ trifluoroacetic acid, and subjected to matrix-assisted \# laser desorption/ionization-time-of-flight (MALDI-TOF) analysis (Ettan MALDI-TOF Pro, GE Healthcare). Spectra were collected from 350 shots per spectrum over an $\mathrm{m} / \mathrm{z}$ range of $600-3000$ and calibrated by two point internal calibration using trypsin auto-digestion peaks ( $\mathrm{m} / \mathrm{z}$ 842.5099, 2211.1046). A peak list was generated using the Ettan MALDI-TOF Pro Evaluation Module (ver 2.0.16). The threshold used for peak-picking was as follows: 5,000 for minimum resolution of monoisotopic mass, 2.5 for $\mathrm{S} / \mathrm{N}$. The search program MASCOT (Mascot Sever 2.3), developed by The Matrixscience (http://www.matrixscience.com) was used for protein identifi- 
cation by PMF. The following parameters were used for the database search: trypsin as the cleaving enzyme, a maximum of one missed cleavage, iodoacetamide (Cys) as a fixed modification, oxidation (Met) as a variable modification, monoisotopic masses, and a mass tolerance of $\pm 0.1 \mathrm{Da}$. MASCOT probability-based MOWSE (molecular weight search) score was calculated for PMF. Protein score was $-10 *$ Log $(\mathrm{P})$, where $\mathrm{P}$ is the probability that the observed match was a random event, and a value $>61$ was considered significant $(p<0.05)$. We made an exception for highly expressed spots and overlapped spots for spot analysis, and accordingly excised one spot which was identified as a single protein.

\section{Immunoblot analysis}

Levels of 4 proteins identified on a 2-DE protein map together with 7 proteins of metabolic importance were confirmed by immunoblot analysis. Tissue lysates were prepared with RIPA buffer (Sigma-Aldrich), homogenized, and centrifuged at $12,000 \times g$ for $20 \mathrm{~min}$. The extract was diluted in $5 \mathrm{X}$ sample buffer $(50 \mathrm{mM}$ Tris of $\mathrm{pH} 6.8,2 \%$ SDS, $10 \%$ glycerol, $5 \% \beta$ mercaptoethanol, and $0.1 \%$ bromophenol blue) and heated for $5 \mathrm{~min}$ at $95^{\circ} \mathrm{C}$ before SDS-polyacrylamide gel electrophoresis (PAGE) using a gel of $6,8,10$, or $12 \%$ acrylamide. After electrophoresis, samples were transferred to a polyvinylidene difluoride (PVDF, Santa Cruz Biotechnology, Santa Cruz, CA, USA) membrane and blocked for $1 \mathrm{~h}$ with TBS (tris-buffered saline)-T buffer (10 mM Tris-HCl, $150 \mathrm{mM} \mathrm{NaCl,} \mathrm{0.1 \%} \mathrm{Tween} 20$ containing 5\% skim milk). The membrane was rinsed in 3 changes of TBS-T buffer, followed by incubation for $3 \mathrm{~h}$ with a 1:1000 dilution of primary polyclonal antibody anti-CK (AB Frontier, Seoul, Korea), anti-HADHA (Invitrogen, Carlsbad, CA, USA) anti- $\beta$-actin, anti-PPAR $\gamma$, anti-UCP1, anti-AMPK, antip-AMPK, anti-CPT1, anti-CES3 (Santa Cruz Biotechnology), anti-FAS, anti-p-ACC, and anti-FAS (Cell Signaling Technology, Beverly, MA, USA) in TBS-T buffer containing 1\% skim milk. After 3 washes, the membrane was incubated for $1 \mathrm{~h}$ with horseradish peroxidase-conjugated anti-goat IgG, anti-mouse IgG, or anti-rabbit IgG secondary antibody (1:1000, AB Frontier) in TBS-T buffer containing 1\% skim milk and developed using enhanced chemiluminescence (ECL; GE Healthcare). Immunoblot analysis was performed by scanning with a UMAX PowerLook 1120 and digitalized using image analysis software (KODAK 1D, Eastman Kodak, Rochester, NY, USA).

\section{Determination of the relative mitochondrial copy} number

Genomic DNA was isolated and purified from $\sim 20 \mathrm{mg}$ of frozen interscapular BAT employing the G-spin Genomic DNA Extraction Kit (Intron). Mitochondrial DNA (mtDNA) content relative to the peroxisome proliferator-activated receptor- $\gamma$ coactivator $1-\alpha$ (PGC1- $\alpha$ ) gene was measured by performing real-time RT-PCR (Stratagene Mx3000p QPCR System, San Diego, CA, USA). Primers for mtDNA were designed using forward 5'-ACA CCAAAA GGA CGA ACC TG3 ' and reverse 5'-ATG GGG AAG AAG CCC TAG AA-3' and for PGC1- $\alpha$ forward 5'-ATG AAT GCA GCG GTC TTA GC-3' and reverse 5'-AAC AAT GGC AGG GTT TGT TC-3' as described previously [46].

\section{Quantitative real-time RT-PCR analysis}

The RNeasy Mini Kit (Qiagen, Valencia, CA, USA) was used for extraction of total RNA from interscapular BAT. Isolated RNA $(1 \mu \mathrm{g})$ was reverse-transcribed using the cDNA Reverse Transcription Kit. Transcript levels of genes were determined by quantitative real-time PCR using SYBR Green I Dye Chemistry, according to the manufacturer's protocol. Primers for UCP1 were designed using forward 5'-GGG ACC TAC AAT GCT TAC AG-3' and reverse 5'-GGT CAT ATG TCA CCA GCT CT-3' and for GAPDH forward 5'-GGT CTC GCT CCT GGAAAG A-3' and reverse 5'-GTA TGA CTC CAC TCA CGG CAA-3'. Reaction mixtures were preheated at $95^{\circ} \mathrm{C}$ for $10 \mathrm{~min}$; temperature conditions were as follows for 45 cycles; melting at $95^{\circ} \mathrm{C}$ for 30 sec, annealing at $60^{\circ} \mathrm{C}$ for $1 \mathrm{~min}$, and elongation at $72^{\circ} \mathrm{C}$ for 1 min. Levels of UCP1 gene were normalized to those of GAPDH.

\section{Statistical analysis}

All experimental results were compared by one-way analysis of variance (ANOVA) using the Statistical Package of Social Science (SPSS, version 14.0K) program; data are expressed as the mean $\pm \mathrm{SD}$. When significant differences between groups were determined $(p<0.05)$, a protected leastsignificant difference (LSD) test, which is a method of multiple comparison consisting of single-step procedures in one-way ANOVA with Tukey's method and Scheffe's method, was used to demonstrate the significant differences between means.

\section{Results}

\section{HFD-induced phenotypes in male and female rats}

Male and female rats were randomly divided into 2 groups, with 20 rats fed an ND and 40 rats fed an HFD, respectively. At the beginning, body weight and food intake of ND and HFD rats were nearly identical in male and female rats. However, after 2 weeks, body weights in HFD-fed rats were significantly higher $(p<0.05)$ than those in their ND-fed counterparts at all subsequent time points in both male and female rats (Fig. 1A). Total body weight gain per food intake of HFD-fed male rats was higher than for females by an average of $\sim 30 \%$ ( $p=$ 0.001) (Fig. 1B). Next, various biochemical parameters were measured in each group (Table 2). Results showed the concentration of plasma HDL-cholesterol was significantly lower in HFD rats, compared with ND rats (males, $p=0.004$; females, $p=0.018$ ), whereas total cholesterol (males, $p=0.032$; females, $p=0.044$ ), TG (males, $p=0.049$; females, $p=0.028$ ), and FFA (males, $p=0.049$ ) levels were significantly higher in HFD rats, compared with those of ND rats in both males and females. In particular, plasma glucose levels were found to be higher in males, compared with female rats (ND, $p=2.2$ $\mathrm{x} 10^{-4}$ ). Levels of plasma insulin and leptin in 7 rats were

Choi/Oh/Choi/Mukherjee/Wang/Liu/Yun 


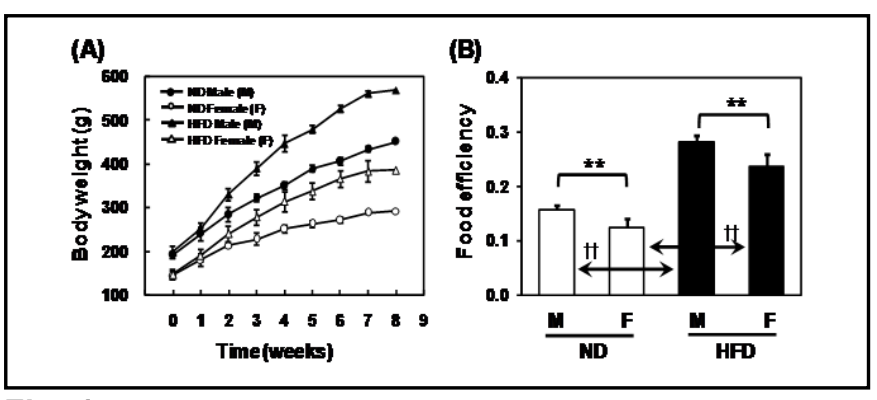

Fig. 1. Body weight (A) and food efficiency (B) in male and female rats fed ND and HFD. Data are presented as mean \pm SD for 7 rats per group and were estimated using the ANOVA test. Statistical significance between male and female rats was determined by a $t$-test, where $p$ value is ${ }^{*} p<0.05$ and ${ }^{* *} p<0.01$, and significance between ND and HFD rats was represented by ${ }^{\dagger} p<0.05$ and ${ }^{\dagger} p<0.01$.

also determined in ND and HFD rats. Results revealed significantly higher average insulin and leptin levels in HFD rats, compared with ND rats (insulin in males, $p=0.03$; leptin in males, $p=1.9 \times 10^{-5}$; leptin in females, $p=0.013$ ), and they were remarkably higher in male rats, compared with female rats (insulin in ND rats, $p=4.1 \times 10^{-4}$; leptin in ND rats, $p=0.011$; leptin in HFD rats, $p=0.007$ ). We also investigated the plasma concentrations of sex hormones, estrogen and testosterone. The concentration of estrogen was significantly higher in HFD rats, compared with ND rats, in both males and females (males, $p=0.01$; females, $p=0.003$ ). However, testosterone concentration was higher in ND rats, compared with HFD rats, only in male rats. These collective results prompted us to perform further proteomic studies.

\section{Proteomic analysis of BAT proteins}

For examination of differential expression of proteins between gender and diet, 2-DE-based proteomic experiments were conducted 3 times using pooled BAT samples from 7 rats per group. In the proteomic study we used a protein concentration of $150 \mu \mathrm{g} / \mathrm{gel}$. BAT proteins were separated by 2-DE using a $\mathrm{pH}$ 3-10 IEF strip for the first dimension and 12\% (w/v) SDS-PAGE gel for the second dimension. Consequently, nearly 595 individual spots were detected, ranging from 6 to $240 \mathrm{kDa}$ mass between $\mathrm{pH} 3$ and 10 (Fig. 2). Based on high scores $(p<0.05)$ and sequence coverage, a total of 55 proteins among $\sim 595$ spots were identified with high confidence by MALDI-TOF/MS and database searches (Table 3).

Gender-dependent expression of BAT proteins in both ND and HFD rats

Image analysis and further statistical analysis allowed detection and identification of 48 proteins whose

\begin{tabular}{lcccc}
\hline Parameter & \multicolumn{2}{c}{ ND } & \multicolumn{2}{c}{ HFD } \\
& Male & Female & Male & Female \\
\hline Glucose $(\mathrm{mg} / \mathrm{ml})$ & $2.08 \pm 0.18^{* * *}$ & $1.64 \pm 0.1^{\dagger}$ & $2.39 \pm 0.24$ & $2.21 \pm 0.58$ \\
TG $(\mathrm{mg} / \mathrm{ml})$ & $0.49 \pm 0.09^{*}$ & $0.47 \pm 0.07^{\dagger}$ & $0.71 \pm 0.23$ & $0.67 \pm 0.19$ \\
Total cholesterol $(\mathrm{mg} / \mathrm{ml})$ & $0.36 \pm 0.16^{*}$ & $0.32 \pm 0.2^{\dagger}$ & $0.6 \pm 0.21$ & $0.54 \pm 0.16$ \\
HDL cholesterol $(\mathrm{mg} / \mathrm{ml})$ & $0.52 \pm 0.11^{* \dagger}$ & $0.51 \pm 0.09^{*}$ & $0.32 \pm 0.11$ & $0.35 \pm 0.12$ \\
Free fatty acid $(\mu \mathrm{mol} / \mathrm{ml})$ & $1.78 \pm 0.2^{\dagger}$ & $1.79 \pm 0.14$ & $2.05 \pm 0.26$ & $1.97 \pm 0.25$ \\
Leptin $(\mathrm{ng} / \mathrm{ml})$ & $6.62 \pm 2.62^{* * *}$ & $2.77 \pm 2.15^{\dagger}$ & $21 \pm 4.28^{* *}$ & $11.2 \pm 6.51$ \\
Insulin $(\mathrm{ng} / \mathrm{ml})$ & $1.67 \pm 0.48^{* * \dagger}$ & $0.57 \pm 0.3$ & $2.43 \pm 0.65$ & $1.5 \pm 1.27$ \\
Estrogen $(\mathrm{ng} / \mathrm{ml})$ & $5.56 \pm 0.55^{* *+\dagger}$ & $8.3 \pm 0.78^{* \dagger}$ & $6.39 \pm 0.47^{* *}$ & $10.83 \pm 1.44$ \\
Testosterone $(\mathrm{ng} / \mathrm{ml})$ & $1.95 \pm 1.85$ & $0.31 \pm 0.07$ & $0.92 \pm 0.56^{* *}$ & $0.35 \pm 0.1$ \\
\hline
\end{tabular}

Table 2. Plasma biochemical parameters in each group. ${ }^{\text {a) }}$ Levels of biochemical parameters were averaged in each group and expressed as a mean $\pm \mathrm{SD}$ of seven separate experiments. ${ }^{1)}$ Statistical significance between male and female rats was determined by a t-test, where $\mathrm{p}$ value is ${ }^{*} p<0.05$ and ${ }^{*} p<0.01$ (male ND vs. female ND in the [ND, Male] column; male HFD vs. female HFD in the [HFD, Male] column). 1) Significance between ND and HFD rats was represented by ${ }^{\dagger} p<0.05$ and ${ }^{\dagger} p<0.01$ (male ND vs. male HFD in the [ND, Male] column; female ND vs. female HFD in the [ND, Female] column).

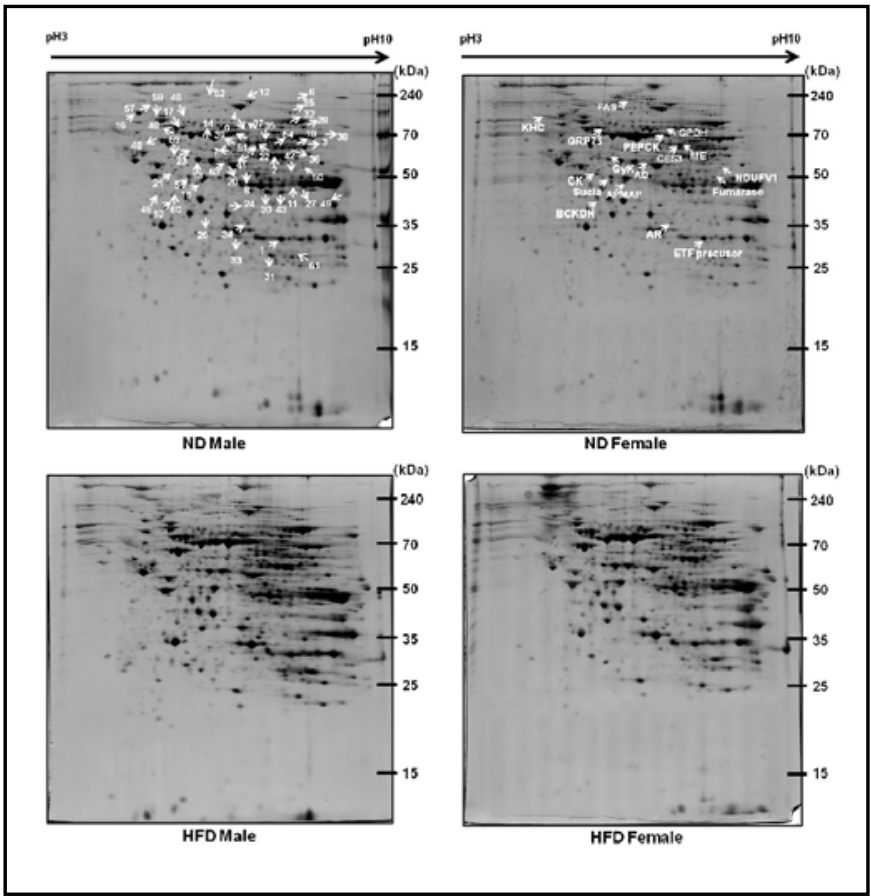

Fig. 2. Representative silver-stained 2-DE gel images of rat BAT proteome in ND and HFD rats. Differentially regulated proteins in each group are marked with arrows and proteins of numbers in gels are listed in Table 3.

expression were significantly modulated in a genderdependent manner in ND or HFD rats. Those proteins were further classified into 5 groups based on their 


\begin{tabular}{|c|c|c|c|c|c|c|c|c|c|}
\hline \multirow{2}{*}{$\begin{array}{l}\text { No. } \\
\text { in gel }\end{array}$} & \multirow[b]{2}{*}{ Protein } & \multirow[b]{2}{*}{ Acc. No. } & \multirow{2}{*}{$\begin{array}{l}\text { Theoretical } \\
\text { (observed) } \\
\text { MW(kDa) }\end{array}$} & \multirow[b]{2}{*}{$\begin{array}{c}\text { Theoretical } \\
\text { pI }\end{array}$} & \multicolumn{2}{|c|}{$\mathrm{ND}^{\mathrm{c})}$} & \multicolumn{2}{|c|}{$\mathrm{HFD}^{\mathrm{c})}$} & \multirow[b]{2}{*}{$\underset{\text { e) }}{\text { Score }}$} \\
\hline & & & & & $\begin{array}{c}\text { Male } \\
\left.(\% \text { vol })^{d}\right)\end{array}$ & $\begin{array}{c}\text { Female } \\
(\% \text { vol })^{\text {d }}\end{array}$ & $\begin{array}{c}\text { Male } \\
(\% \text { vol })\end{array}$ & $\begin{array}{c}\text { Female } \\
\left.(\% \text { vol })^{d}\right)\end{array}$ & \\
\hline
\end{tabular}

Group I : Protein showed gender_difference which are higher levels in males

1 electron transfer flavoprotein subunit alpha gi|57527204 35 (31) precursor (ETF precursor) 8.62 $08+0.13$

$17 \quad$ grp 75

19 methylcrotonoyl-CoA carboxylase beta g) gi 40018616 $61(65)$ gi 1000439
gi 58865926 $73(75)$ 6.23 chain (MCC-beta chain)

22 NADP-dependent malic enzyme (ME)

34 aldose reductase (AR)

39 asparagine synthetase (AS)

41 cytosol aminopeptidase (CAP)

43 zinc-binding alcohol dehydrogenase domain-containing protein 2 (ZADH2)

$50 \quad$ NADH dehydrogenase (ubiquinone) flavoprotein 1(NDUFV1)

54 perilipin-1 (PLIN)

$\begin{array}{ll}\text { gi } 266504 & 64(63) \\ \text { gi } 6978491 & 36(34) \\ \text { gi } 148747576 & 64(60) \\ \text { gi } 58865398 & 56(57) \\ \text { gi } 157817316 & 40(40) \\ \text { gi } 149061921 & 36(46) \\ \text { gi } 6981372 & 55(58)\end{array}$

5.87

8.56

6.49

6.26

6.01

6.77

7.63

5.89

6.37

6.37

$0.13 \pm 0.01$

$0.10 \pm 0.00$

$0.25 \pm 0.01$

$0.12 \pm 0.01$

$0.67 \pm 0.06^{\mathrm{cc}}$

$0.10 \pm 0.01^{\mathrm{c}}$

$0.14 \pm 0.01^{\mathrm{cc}}$

$0.11 \pm 0.00^{\text {cc }}$

$0.18 \pm 0.04 \quad 0.10 \pm 0.01^{\mathrm{a}}$

$0.06 \pm 0.01^{\text {aa }}$

$0.19 \pm 0.02^{\mathrm{a}}$

$0.08 \pm 0.01^{\mathrm{a}}$

$0.53 \pm 0.05^{\mathrm{a}}$

$0.06 \pm 0.00^{\mathrm{a}}$

$0.11 \pm 0.00^{\text {aa }}$

$0.10 \pm 0.00^{\text {aa }}$

$0.10 \pm 0.01$

Group II : Protein showed gender difference which are higher levels in females

5 sucla2 protein

10 branched chain keto acid dehydrogenase El (BCKDH)

16 kinesin heavy chain isoform $5 \mathrm{C}$ (KHC)

18 adipocyte plasma membrane-associated protein (APMAP)

21 creatine kinase B-type (CK B-type)

23 glycerol kinase (GyK)

37 glycerol-3-phosphate dehydrogenase 2 (GPDH)

42 fumarase

45 zinc binding alcohol dehydrogenase, domain containing 1 (ZADHl)

58 NADH dehydrogenase (ubiquinone) Fe-S protein 1 (NDUFS1)

60 alpha-enolase isoform 1 (ENO1)

$3845496-70(70)$

gi|187469277 $47.7(43)$

gi|165971320 $43(40)$

6.09

$0.15 \pm 0.01$

$\begin{array}{ll}6.06 & 0.19 \pm 0.02\end{array}$

6.41

$0.09 \pm 0.03$

gi|157819777 $109(101)$
gi| 77735352
42 (42)

5.86

$0.01 \pm 0.1$

\section{gi|31542401 $42(43)$}

gil $158186653 \quad 58(54)$

5.64

$0.09 \pm 0.02$

5.33

$0.07 \pm 0.04$

gi|149047794 $66(72)$

5.49

$0.04 \pm 0.01^{\mathrm{c}}$

$0.08 \pm 0.01^{\mathrm{c}}$

gi|227665 54 (44)

gi| $149025122 \quad 22(37)$

9.14

$0.07 \pm 0.01^{\mathrm{cc}}$

gil149046009 74 (78)

7.67

$0.05 \pm 0.01$

$5.74 \quad 0.08 \pm 0.02$

$0.06 \pm 0.01^{a}$

$0.11 \pm 0.00^{\mathrm{a}}$

$0.13 \pm 0.01$

89

gi|158186649 $47(40)$

6.16

$0.02 \pm 0.00$

$0.27 \pm 0.02^{\text {aа }}$

$0.18 \pm 0.04^{\mathrm{b}}$

$0.30 \pm 0.04$

$0.36 \pm 0.05$

$0.29 \pm 0.02^{\mathrm{a}}$

$0.17 \pm 0.02^{\text {a }}$

$0.24 \pm 0.02^{b}$

$0.10 \pm 0.02^{b}$

$0.18 \pm 0.03$

321

$0.08 \pm 0.01^{\text {aa }}$

$0.14 \pm 0.01^{\mathrm{a}}$

$0.01 \pm 0.00^{\text {b }}$

$0.06 \pm 0.01$

$0.14 \pm 0.01$

$0.17 \pm 0.04^{\mathrm{a}}$

$0.07 \pm 0.01^{2}$

$0.11 \pm 0.01^{2}$

$0.11 \pm 0.04^{b}$

$0.06 \pm 0.01^{\mathrm{b}}$

$0.05 \pm 0.01^{\mathrm{b}}$

$0.21 \pm 0.01$

$0.09 \pm 0.01 \quad 176$

$0.08 \pm 0.00^{\mathrm{d}} \quad 243$

$0.09 \pm 0.0 \mathrm{l}^{\mathrm{a}}$

$0.11 \pm 0.01^{\mathrm{aa}}$

$0.13 \pm 0.01^{\mathrm{b}}$

$0.16 \pm 0.00^{\mathrm{d}}$

$0.04 \pm 0.01^{\mathrm{b}}$

$0.06 \pm 0.00^{\mathrm{d}}$

90

$0.14 \pm 0.01^{a}$

$0.11 \pm 0.00^{\mathrm{bb}}$

$0.12 \pm 0.00$

132

Group III: Protein showed gender difference both in ND or HFD rats with opposite patterns

51 carboxylesterase 3 (CES3)

52 fatty acid synthase (FAS)

53 aldehyde dehydrogenase (ADH)

55 rCG55067

gi| $57013350 \quad 62(60) \quad 6.10$

gi $2506136 \quad 275(276) \quad 5.96$

gi| $16073616 \quad 48(50) \quad 6.06$

$0.07 \pm 0.03$

$0.03 \pm 0.01^{\mathrm{c}}$

$0.08 \pm 0.00^{\circ}$

$0.07 \pm 0.00^{\text {aa }}$

$0.01 \pm 0.00^{b}$

$0.03 \pm 0.01^{\mathrm{dd}}$

62

$0.16 \pm 0.02^{\mathrm{a}}$

$0.05 \pm 0.00^{\mathrm{aa}}$

$0.07 \pm 0.00^{\mathrm{a}}$

$0.09 \pm 0.01^{\mathrm{a}}$

$0.023 \pm 0.02^{b}$

$0.05 \pm 0.01^{b}$

$0.03 \pm 0.01^{\mathrm{b}}$

$0.11 \pm 0.00^{\mathrm{bh}}$

$0.17 \pm 0.02 \quad 89$

$0.03 \pm 0.01^{\mathrm{d}} \quad 93$

$0.07 \pm 0.001 \quad 80$

$0.13 \pm 0.00^{\mathrm{dd}} \quad 76$

Group IV: Proteins showing a gender-difference in the regulation patterns in only ND or HFD rats

$$
\text { (DLD precursor) }
$$

3 M2 pyruvate kinase -like isoform 1

(PKM2-like isoform 1)

6 acly protein

7 T-complex protein 1 subunit beta (TPSB)

8 tu translation elongation factor (EFTu) $\begin{array}{llll}\text { gi|} 40786469 & 54(54) & 7.96 & 0.73 \pm 0.06\end{array}$

gi|293350511 $58(59)$

gi|71680955 $120(121)$

gi| 54400730

gi 157820845
$57(57)$

$44(44)$

\subsection{5}

\subsection{3}

6.01

7.65
$0.56+0.05$

\section{$0.12 \pm 0.03^{\mathfrak{c}}$}

$0.12 \pm 0.04$

$0.10 \pm 0.01^{\mathrm{c}}$
$0.66+0.09$

$0.32 \pm 0.03^{\text {aa }}$

$0.06 \pm 0.01^{\mathrm{a}}$

$0.10 \pm 0.04$

$0.08 \pm 0.01^{\mathrm{a}}$
$0.83 \pm 0.05^{\mathrm{b}}$

$0.71 \pm 0.05$

$0.57 \pm 0.06$

$0.21 \pm 0.04$

$0.16 \pm 0.01^{\mathrm{b}}$

$0.06 \pm 0.02$
$0.47 \pm 0.08^{\text {dd }} 163$

$0.15 \pm 0.04^{\mathrm{d}} \quad 331$

$0.10 \pm 0.02 \quad 221$

$0.06 \pm 0.01 \quad 116$ expression patterns in response to diet and gender (Table 4). Groups I and II contained proteins showing genderdifference with identical expression patterns in both ND and HFD rats. Of these, levels of 11 proteins were higher in males (Group I), while those of 12 proteins were higher in females (Group II). Highly regulated proteins in males in both ND and HFD rats included the following: electron-transfer flavoprotein (ETF), NADP-dependent malic enzyme (ME), asparagine synthase (AS), NADH dehydrogenase (ubiquinone) flavoprotein 1 (NDUFV1), zinc-binding alcohol dehydrogenase domain-containing protein 2 (ZADH2), glucose-regulated

Choi/Oh/Choi/Mukherjee/Wang/Liu/Yun 


\begin{tabular}{|c|c|c|c|c|c|c|c|c|c|}
\hline \multirow{2}{*}{$\begin{array}{l}\text { No. } \\
\text { in gel }\end{array}$} & \multirow[b]{2}{*}{ Protein } & \multirow[b]{2}{*}{ Acc. No. } & \multirow{2}{*}{$\begin{array}{l}\text { Theoretical } \\
\text { (observed) } \\
\mathrm{MW}(\mathrm{kDa})\end{array}$} & \multirow[b]{2}{*}{$\begin{array}{c}\text { Theoretical } \\
\text { pI }\end{array}$} & \multicolumn{2}{|c|}{$\mathrm{ND}^{\mathrm{c})}$} & \multicolumn{2}{|c|}{$\mathrm{HFD}^{\mathrm{c})}$} & \multirow[b]{2}{*}{$\begin{array}{c}\text { Score } \\
\text { e) }\end{array}$} \\
\hline & & & & & $\begin{array}{c}\text { Male } \\
(\% \text { vol })^{d)} \\
\end{array}$ & $\begin{array}{l}\text { Female } \\
\left.(\% \text { vol })^{d}\right)\end{array}$ & $\begin{array}{c}\text { Male } \\
(\% \text { vol })^{d}\end{array}$ & $\begin{array}{l}\text { Female } \\
\left.(\% \text { vol })^{d}\right)\end{array}$ & \\
\hline 11 & $\begin{array}{l}\text { pyruvate dehydrogenase E1 component } \\
\text { subunit alpha (PDA) }\end{array}$ & gi|109503594 & $44(42)$ & 8.49 & $0.08 \pm 0.00$ & $0.06 \pm 0.01^{\mathrm{a}}$ & $0.11 \pm 0.02$ & $0.09 \pm 0.03$ & 133 \\
\hline 12 & pyruvate carboxylase $(\mathrm{PC})$ & gi|929988 & $130(130)$ & 6.25 & $0.15 \pm 0.05$ & $0.27 \pm 0.04^{\mathrm{a}}$ & $0.14 \pm 0.04$ & $0.14 \pm 0.04^{d}$ & 344 \\
\hline 13 & enolase 3 (ENO3) & gi| 109468300 & $54(50)$ & 5.81 & $0.04 \pm 0.02$ & $0.05 \pm 0.02$ & $0.06 \pm 0.01^{b}$ & $0.09 \pm 0.01^{\mathrm{d}}$ & 101 \\
\hline 14 & epoxide hydrolase 2 (sEH2) & gi|55716049 & $62(61)$ & 5.76 & $0.06 \pm 0.02$ & $0.08 \pm 0.01$ & $0.07 \pm 0.01^{b}$ & $0.04 \pm 0.00$ & 195 \\
\hline 20 & N-acetylgalactosamine kinase (NAGK) & gi|62078569 & $51(47)$ & 6.04 & $0.02 \pm 0.00^{\mathrm{c}}$ & $0.02 \pm 0.00$ & $0.05 \pm 0.00^{b}$ & $0.02 \pm 0.00$ & 90 \\
\hline 27 & $\begin{array}{l}\text { isocitrate dehydrogenase [NAD] subunit } \\
\text { beta precursor(ICDH subunit beta precursor) }\end{array}$ & gi|55926203 & $42(41)$ & 8.89 & $0.23 \pm 0.03^{\mathrm{c}}$ & $0.24 \pm 0.03^{\mathrm{a}}$ & $0.11 \pm 0.04$ & $0.10 \pm 0.00^{\mathrm{d}}$ & 167 \\
\hline 35 & EH domain-containing protein 1 (EHD1) & gi||169642494 & $62(62)$ & 6.46 & $0.22 \pm 0.00^{\mathrm{c}}$ & $0.18 \pm 0.01^{\mathrm{a}}$ & $0.19 \pm 0.01$ & $0.16 \pm 0.05$ & 114 \\
\hline 36 & $\begin{array}{l}\text { acyl-CoA synthetase family member } 2 \\
\text { precursor (ACSF2 precursor) }\end{array}$ & gi| 77993368 & $68(60)$ & 8.39 & $0.19 \pm 0.03^{\mathrm{c}}$ & $0.12 \pm 0.01^{\mathrm{a}}$ & $0.11 \pm 0.01$ & $0.11 \pm 0.03$ & 153 \\
\hline 38 & acyl-Coenzyme A dehydrogenase (ACD) & gi|56541110 & $71(67)$ & 8.94 & $0.20 \pm 0.03$ & $0.11 \pm 0.02^{\mathrm{a}}$ & $0.27 \pm 0.04$ & $0.22 \pm 0.00^{\mathrm{dd}}$ & 176 \\
\hline 40 & $\begin{array}{l}\text { dihydrolipoyllysine-residue } \\
\text { acetyltransferase component of pyruvate } \\
\text { dehydrogenase complex (ACEF) }\end{array}$ & gi|78365255 & $67(69)$ & 8.76 & $0.15 \pm 0.01$ & $0.22 \pm 0.02^{\mathrm{a}}$ & $0.17 \pm 0.03$ & $0.17 \pm 0.03$ & 85 \\
\hline 46 & G elongation factor (EF-G) & gi|293345129 & $86(89)$ & 6.07 & $0.02 \pm 0.00$ & $0.04 \pm 0.00^{\mathrm{aaa}}$ & $0.03 \pm 0.01$ & $0.02 \pm 0.00^{\mathrm{dd}}$ & 203 \\
\hline 48 & unnamed protein product (PY02383) & gi|1334284 & $58(62)$ & 5.35 & $0.07 \pm 0.01^{\mathrm{ci}}$ & $0.14 \pm 0.03^{\mathrm{a}}$ & $0.15 \pm 0.01$ & $0.15 \pm 0.07$ & 88 \\
\hline 49 & $\begin{array}{l}\text { isocitrate dehydrogenase } 3 \text { (NAD) gamma } \\
\text { (ICDH3 gamma) }\end{array}$ & gi|1 149029908 & $41(40)$ & 9.08 & $0.09 \pm 0.01$ & $0.05 \pm 0.01^{\mathrm{a}}$ & $0.14 \pm 0.04$ & $0.21 \pm 0.03^{\mathrm{d}}$ & 87 \\
\hline 57 & heat shock $70 \mathrm{kDa}$ protein 4 (HSP70RY) & gi|24025637 & $94(103)$ & 5.13 & $0.03 \pm 0.01^{\mathrm{c}}$ & $0.06 \pm 0.01^{\mathrm{a}}$ & $0.05 \pm 0.00$ & $0.05 \pm 0.00$ & 178 \\
\hline 59 & cytosolic non-specific dipeptidase (CNDP2) & gi|58219062 & $53(52)$ & 5.43 & $0.04 \pm 0.02^{\mathrm{c}}$ & $0.08 \pm 0.0 \mathrm{l}^{\mathrm{a}}$ & $0.08 \pm 0.00$ & $0.07 \pm 0.02$ & 236 \\
\hline 61 & adenylate kinase 2 (AK2) & gil 77020256 & $25(28)$ & 7.01 & $0.06 \pm 0.03^{\mathrm{c}}$ & $0.05 \pm 0.03$ & $0.17 \pm 0.04^{b}$ & $0.09 \pm 0.01$ & 64 \\
\hline \multicolumn{10}{|c|}{ Group V: Proteins that were HFD-responsive, but not expressed in a gender-dependent } \\
\hline 24 & UDP-glucose 4-epimerase (GALE) & gi| 162287387 & $38(37)$ & 6.02 & $0.09 \pm 0.02^{\mathrm{c}}$ & $0.10 \pm 0.03$ & $0.03 \pm 0.01$ & $0.03 \pm 0.01^{\mathrm{dd}}$ & 68 \\
\hline 26 & malate dehydrogenase (MHD) & gi 15100179 & $36(36)$ & 6.16 & $0.22 \pm 0.02^{\mathrm{c}}$ & $0.23 \pm 0.02$ & $0.11 \pm 0.04$ & $0.10 \pm 0.00^{\mathrm{d}}$ & 93 \\
\hline 28 & $\begin{array}{l}\text { mitochondrial long-chain enoyl-CoA } \\
\text { hydratase/3-hydroxycyl-CoA } \\
\text { dehydrogenase alpha-subunit (HADHA) }\end{array}$ & gi 510108 & $83(76)$ & 9.11 & $0.36 \pm 0.12^{\mathrm{c}}$ & $0.18 \pm 0.03$ & $0.06 \pm 0.04$ & $0.06 \pm 0.01^{\mathrm{dd}}$ & 180 \\
\hline 30 & $\begin{array}{l}\text { short-chain specific acyl-CoA } \\
\text { dehydrogenase (SCAD) }\end{array}$ & gi $\mid 11968090$ & $45(41)$ & 8.47 & $0.43 \pm 0.05^{\mathrm{c}}$ & $0.39 \pm 0.06$ & $0.55 \pm 0.05$ & $0.59 \pm 0.09^{d}$ & 145 \\
\hline 31 & phosphoglycerate mutase 1(PGM1) & gi 114326546 & $28(28)$ & 6.67 & $0.23 \pm 0.04^{\mathrm{c}}$ & $0.20 \pm 0.02$ & $0.13 \pm 0.03$ & $0.13 \pm 0.01^{\mathrm{d}}$ & 172 \\
\hline 32 & serotransferrin precursor ( $\mathrm{TF}$ precursor) & gi 61556986 & $78(79)$ & 7.14 & $0.07 \pm 0.02^{\mathrm{cc}}$ & $0.06 \pm 0.01$ & $0.21 \pm 0.01$ & $0.21 \pm 0.03^{\mathrm{dd}}$ & 297 \\
\hline 33 & $\begin{array}{l}\text { voltage-dependent anion-selective channel } \\
\text { protein } 2 \text { (VDAC2) }\end{array}$ & gi 13786202 & $32(31)$ & 7.44 & $0.14 \pm 0.04^{\mathrm{c}}$ & $0.14 \pm 0.04$ & $0.03 \pm 0.01$ & $0.05 \pm 0.01^{\mathrm{d}}$ & 79 \\
\hline
\end{tabular}

Table 3. Differentially regulated and major BAT proteins in male and female rats identified through proteomic analysis ${ }^{\text {a) No. in }}$

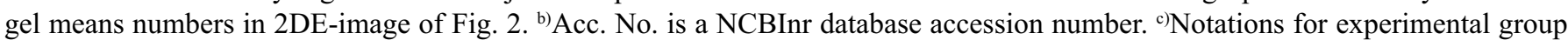
division: LFD, control rats fed LFD; HFD, rats fed HFD. ${ }^{d}$ Statistical significance was determined by a t-test, where p-values is ${ }^{*} p<0.05$ and ${ }^{* *} p<0.01$; Nor Male vs. Nor Female ${ }^{\mathrm{a}} p<0.05$ and ${ }^{\text {aa }} p<0.01$; HF Male vs. HF Female ${ }^{\mathrm{b}} p<0.05$ and ${ }^{\mathrm{bb}} p<0.01$; Nor Male vs. HF Male ${ }^{\mathrm{c}} p<0.05$ and ${ }^{\mathrm{cc}} p<0.01$; Nor Female vs. HF Female ${ }^{\mathrm{d}} p<0.05$ and ${ }^{\mathrm{dd}} p<0.01$. For each protein, the relative intensity was

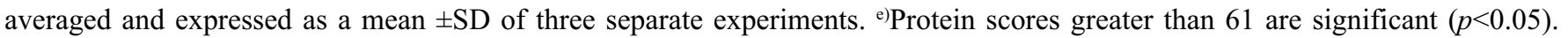

protein 75 (GRP75), cytosol aminopeptidase (CAP), methylcrotonoyl-CoA carboxylase beta chain (MCC-beta chain), T-complex protein 1 subunit gamma (TPSG), aldose reductase (AR), and perilipin-1 (PLIN) (Fig. 3 and Table 3).

Meanwhile, higher protein levels in females of both ND and HFD rats included the following: glycerol kinase (GyK), glycerol-3-phosphate dehydrogenase (GPDH), phosphoenolpyruvate carboxylase (PEPCK), succinylCoA ligase subunit beta 2, mitochondrial (Sucla2),

Gender Differences in Brown Adipose Tissues of Rats fumarase, branched chain $\alpha$-keto acid dehydrogenase complex (BCKADC), creatine kinase (CK), adipocyte plasma membrane-associated protein (APMAP), kinesin heavy chain isoform 5C (KHC), NADH dehydrogenase (ubiquinone) $\mathrm{Fe}-\mathrm{S}$ protein 1(NDUFS1), zinc binding alcohol dehydrogenase domain-containing protein 1 (ZADH1), and enolase 1(ENO1) (Fig. 4 and Table 3).

Among those proteins, $\mathrm{KHC}$ exhibited the greatest gender difference in both ND and HFD rats. Of particular interest, 4 proteins showed gender-differences with 
opposite expression patterns in both ND and HFD rats (Group III); these included fatty acid synthase (FAS), carboxylesterase 3 (CES3), aldehyde dehydrase (ADH), and rCG55067 (Fig. 5). In addition, and of particular interest, as shown in Fig. 6 and 7, and Table 3, 21 proteins showed gender difference only in ND or HFD (Group IV), while 7 showed gender-dependent expression only in HFD rats.

BAT proteins that were HFD-responsive, but not expressed in a gender-dependent manner

As shown in Fig. 7 and Table 3, we also found that a total of 7 proteins showed no gender-difference, but exhibited significant HFD-responsive regulation in both males and females. Of these, levels of 2 proteins were higher in HFD-fed rats, whereas those of 5 proteins were markedly lower in HFD rats, compared with ND rats.

\section{Validation of results from proteomic analysis} using immunoblot analysis

Although our proteomic data indicated differential protein expression between male and female rats, we could not exclude the possibility of technical errors and artificial effects in proteomic analysis. To address this issue, levels of 4 proteins of interest (CK, FAS, CES3, and HADHA) together with 7 metabolically important proteins in BAT (e.g. PPAR $\gamma$, GLUT4, UCP1, CPT1, AMPK, p-AMPK, and p-ACC) were investigated by immunoblot analysis. As shown in Fig. 8, protein levels of 4 proteins were in line with those from proteomic analysis showing gender- or HFD-responsive regulation. Levels of UCP1 and 6 other proteins of metabolic importance also showed significant gender-differences and showed HFD responsive regulation patterns (Fig. 9 and Fig. 10).

Mitochondrial content, $\mathrm{PGC} 1-\alpha, \mathrm{UCP} 1$ protein, and mRNA levels of UCP1 in BAT of ND and HFD-fed rats were determined for comparison of thermogenic activity. As shown in Fig. 10, all were higher in females than in males under both dietary conditions.

\section{Discussion}

In the present study, using the hypothesis that members of the BAT proteins can be either directly or indirectly related to control of body weight in both genders, we performed comparative proteome analysis in BAT comparing male and female rats for response to HFD treatment. A variety of studies have raised the possibility of the existence of sex dimorphism in metabolic activity

\begin{tabular}{lcccc}
\hline Protein ${ }^{\text {a) }}$ & ND(F)/ND(M) & HFD(F)/HFD(M) & $\begin{array}{r}\text { HFD(M)/ } \\
\text { ND(M) }\end{array}$ & $\begin{array}{r}\text { HFD(F) } \\
\text { ND(F) }\end{array}$ \\
\hline Group I : Proteins showing gender differences with higher levels in males \\
ETF precursor & -1.33 & -1.32 & -1.05 & -1.04 \\
TPSG & -1.67 & -1.43 & 1.00 & 1.17 \\
Grp75 & -1.32 & -2.00 & 1.04 & -1.46 \\
MCC-beta chain & -1.50 & -1.63 & 1.08 & 1.00 \\
ME & -1.26 & -1.28 & -1.63 & -1.66 \\
AR & -1.67 & -1.67 & 1.50 & 1.50 \\
AS & -1.27 & -1.43 & -1.40 & -1.57 \\
CAP & -1.10 & -1.40 & 1.27 & 1.00 \\
ZADH2 & -1.80 & -2.00 & -1.50 & -1.67 \\
NDUFV1 & -1.50 & -3.00 & -1.50 & -3.00 \\
PLIN & -1.18 & -1.30 & 1.00 & -1.10 \\
Group II : Proteins showing gender differences with higher levels in females \\
PEPCK & 1.80 & 1.67 & 1.20 & 1.11 \\
Sucla2 protein & 1.53 & 1.50 & 1.26 & 1.24 \\
BCKDH & 1.89 & 1.80 & 1.11 & 1.06 \\
KHC & 8.00 & 2.00 & 3.00 & -1.33 \\
APMAP & 1.56 & 1.75 & -1.13 & 1.00 \\
CK -B-type & 2.43 & 1.91 & 1.57 & 1.24 \\
GyK & 1.75 & 1.50 & 1.50 & 1.29 \\
GPDH & 1.38 & 1.60 & -1.60 & -1.38 \\
Fumarase & 1.29 & 1.23 & 1.86 & 1.78 \\
ZADH1 & 2.20 & 1.50 & -1.25 & -1.83 \\
NDUFS1 & 1.75 & 1.09 & 1.38 & -1.17 \\
ENO1 & 3.50 & 3.00 & -2.00 & -2.33 \\
Group IIt Protein showing & & &
\end{tabular}

Group III: Protein showing gender differences both in ND and HFD rats with opposite regulation patterns

$\begin{array}{lcccc}\text { with opposite regulation patterns } & & & \\ \text { CES3 } & 2.29 & -1.35 & 3.29 & 1.06 \\ \text { FAS } & 1.67 & -1.67 & 1.67 & -1.67 \\ \text { ADH } & -1.14 & 2.33 & -2.67 & 1.00 \\ \text { rCG55067 } & -1.22 & 1.18 & 1.00 & 1.44\end{array}$

Group IV: Proteins showing gender differences only in either ND or HFD rats $\begin{array}{lllll}\text { DLD precursor } & -1.11 & -1.17 & 1.14 & 1.08\end{array}$ $\begin{array}{lllll}\text { PKM2-like isoform } 1 & -1.75 & -1.21 & 1.02 & 1.47\end{array}$

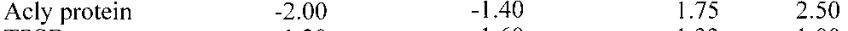
$\begin{array}{lllll}\text { TPSB } & -1.20 & -1.60 & 1.33 & 1.00\end{array}$

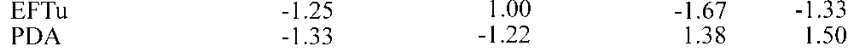
$1.00-1.07 \quad-1.93$ 1.50 $\begin{array}{lllll}\text { SEH2 } & 1.33 & -1.75 & 1.17 & -2.00 \\ \text { NAGK } & 1.00 & -2.50 & 2.50 & 1.00\end{array}$

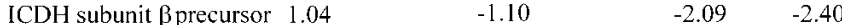
$\begin{array}{lllll}\text { EHD1 } & -1.22 & -1.19 & -1.16 & -1.13\end{array}$

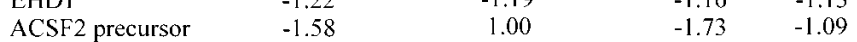
$\begin{array}{lrrrr}\mathrm{ACD} & -1.82 & -1.23 & -1.73 & -1.09 \\ \mathrm{ACEF} & 1.47 & 1.00 & 1.13 & -1.29\end{array}$ $\begin{array}{lrrrr}\text { ACEF } & 1.47 & 1.00 & 1.13 & -1.29 \\ \text { EF-G } & 2.00 & -1.50 & 1.50 & -2.00\end{array}$ PY02383 200 $\begin{array}{lrrrr}\text { ICDH3 gamma } & -1.80 & 1.50 & 1.56 & 4.20\end{array}$ $\begin{array}{lllll}\text { HSP70RY } & 2.00 & 1.00 & 1.67 & -1.20\end{array}$ $\begin{array}{lllll}\text { CNDP2 } & 2.00 & -1.14 & 2.00 & -1.14\end{array}$ $\begin{array}{lllll}\text { AK2 } & -1.20 & -1.89 & 2.83 & 1.80\end{array}$

Group V: Proteins that were HFD-responsive, but not expressed in a gender-dependent manne

$\begin{array}{lllll}\text { GALE } & 1.11 & 1.00 & -3.00 & -3.33\end{array}$ $\begin{array}{llll}1.05 & -1.10 & -3.00 & -3.33 \\ -2.00 & 1.00 & -6.00 & -3.00\end{array}$ $\begin{array}{lllll}\text { HADHA } & -2.00 & 1.00 & -6.00 & -3.00\end{array}$ $\begin{array}{llllr}\text { SCAD } & -1.10 & 1.07 & 1.28 & 1.51\end{array}$ $\begin{array}{lrrrr}\text { PGM1 } & -1.15 & 1.00 & -1.77 & -1.54 \\ \text { TF precursor } & -1.17 & 1.00 & 3.00 & 3.50\end{array}$ \begin{tabular}{lllll} 
VDAC2 & 1.00 & 1.67 & -4.67 & -2.80 \\
\hline
\end{tabular}

Table 4. Fold changes of differentially expressed BAT protein

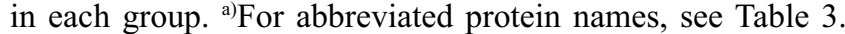

in adipose tissues, such as energy expenditure and oxidative capacity $[18,21,35,36]$. Greater oxidative capacity with more effective antioxidant machinery in female rats, compared to males, has been demonstrated. The increased oxidative activity may result in higher energy intake but lower body fat deposition, compared with males [34]. Compared to male rats, female rats have higher protein content (particularly UCP1), and greater respiratory and phosphorylative capacities in mitochondria, thereby resulting in higher energy expenditure $[18,35$,

Choi/Oh/Choi/Mukherjee/Wang/Liu/Yun 
Fig. 3. Differentially expressed BAT proteins showing gender difference in both ND and HFD rats with identical patterns. These proteins showed higher expression in males. Data are exhibited as mean values \pm SD of volume density (\%) of the changed spot in 3 individual gels using pooled BAT from 7 rats per group. These 11 proteins have $p$ values $<0.05$ when comparing males and females fed ND and/or HFD. Statistical significance between male (M) and female (F) rats was determined by a $t$-test, where $p$ values were ${ }^{*} p<0.05$ and ${ }^{* *} p<0.01$ and significance between ND and HFD rats was represented by ${ }^{\dagger} p<0.05$ and ${ }^{\prime \prime} p<0.01$. For abbreviation of each protein name, see Table 3. Arabic numerals in parenthesis of bar graphs indicate spot numbers in zoon-in gel images.

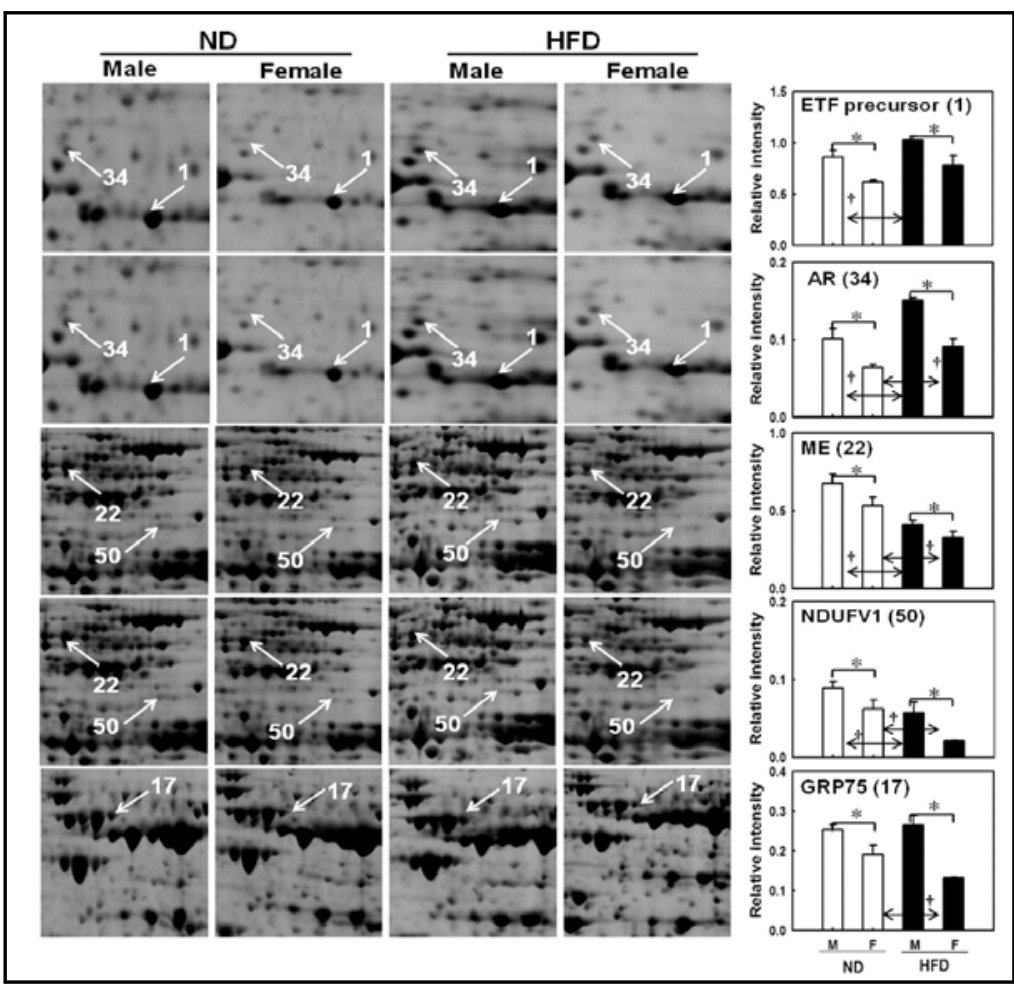

36]. Borrás and coworkers [47] also found that females exhibited higher antioxidant gene expression and lower oxidative damage than males. Although they did not link their findings to obesity, these results also appear to be responsible for higher fat oxidation in females. During the post-cafeteria period, BAT was found to be more efficiently activated in females who experienced higher body weight loss than in males after removal of the cafeteria diet [15]. Greater sensitivity of female rats to cold, compared with male rats, has been demonstrated, and resistance to cold is a physiological priority [16]. Female rats are known to be more sensitive to cold because their threshold temperature for the thermogenesis response is set at a higher value $\left(22^{\circ} \mathrm{C}\right)$ than that of males $\left(18^{\circ} \mathrm{C}\right)$. Female rats might have a low threshold temperature for cold-induced thermogenic response. In other words, their BAT is already activated at $22^{\circ} \mathrm{C}$, whereas male rats are not (or less) sensitive to cold at this temperature [20]. Male and female animals acclimated to $22^{\circ} \mathrm{C}$ have different specific UCP1 levels in BAT, which were double for female rats, compared with male rats [20].

However, several conflicting results suggesting higher thermogenic activity in males than females have been reported. For example, higher levels of $\beta_{3}$ adrenoceptor in male rats, compared with females, have been demonstrated, pointing to greater thermogenic capacity and less weight gain in males [21]. In addition, female rats appeared to have decreased energy expenditure through protection of metabolically active organs to a greater extent than male rats during calorie restriction [22]. Rodríguez and Palou [15] demonstrated a gender difference in cold, diet, and overweight-induced stimulation of expression of UCP1 in BAT. Upon chronic cafeteria diet feeding, female rats have been shown to attain a larger excess of body weight than males. BAT of female lean control rats has been shown to be already activated, compared to that of males [15]. Taken together, it remains to be determined whether males or females have higher innate thermogenic activity leading to suppression of weight gain when exposed to HFD.

In the current study, we found numerous proteins showing differential expression in a gender-dependent manner at 8 weeks of breeding duration. For interpretation of the results of proteomic analysis, we categorized identified proteins into 5 groups (Table 3): Groups I and II contained proteins showing gender difference in both ND and HFD rats with identical patterns. The levels of some proteins were higher in males (Group I), whereas some were higher in females (Group II). Group III included proteins showing gender-difference in both ND and HFD rats with opposite patterns. Group IV proteins were defined as ones that showed a gender-difference in the regulation patterns in only ND or HFD rats. Lastly, proteins categorized into Group V showed no genderdifference but did show a HFD response. Due to space limitation, a limited number of proteins showing a clear gender difference were discussed here.

Cell Physiol Biochem 2011;28:933-948 941 
Fig. 4. Differentially expressed BAT proteins showing gender difference in both ND and HFD rats with identical patterns. These proteins showed higher expression in females. Data are exhibited as mean values $\pm \mathrm{SD}$ of volume density (\%) of the changed spot in 3 individual gels using pooled BAT from 7 rats per group. These 12 proteins have $p$ values $<0.05$ when comparing males and females fed ND and/or HFD. Statistical significance between male (M) and female (F) rats was determined by a $t$ test, where $p$ values were ${ }^{*} p<0.05$ and ${ }^{* *} p<0.01$ and significance between ND and HFD rats was represented by ${ }^{\dagger} p<0.05$ and ${ }^{\dagger} p<0.01$. For abbreviation of each protein name, see Table 3. Arabic numerals in parenthesis of bar graphs indicate spot numbers in zoon-in gel images.

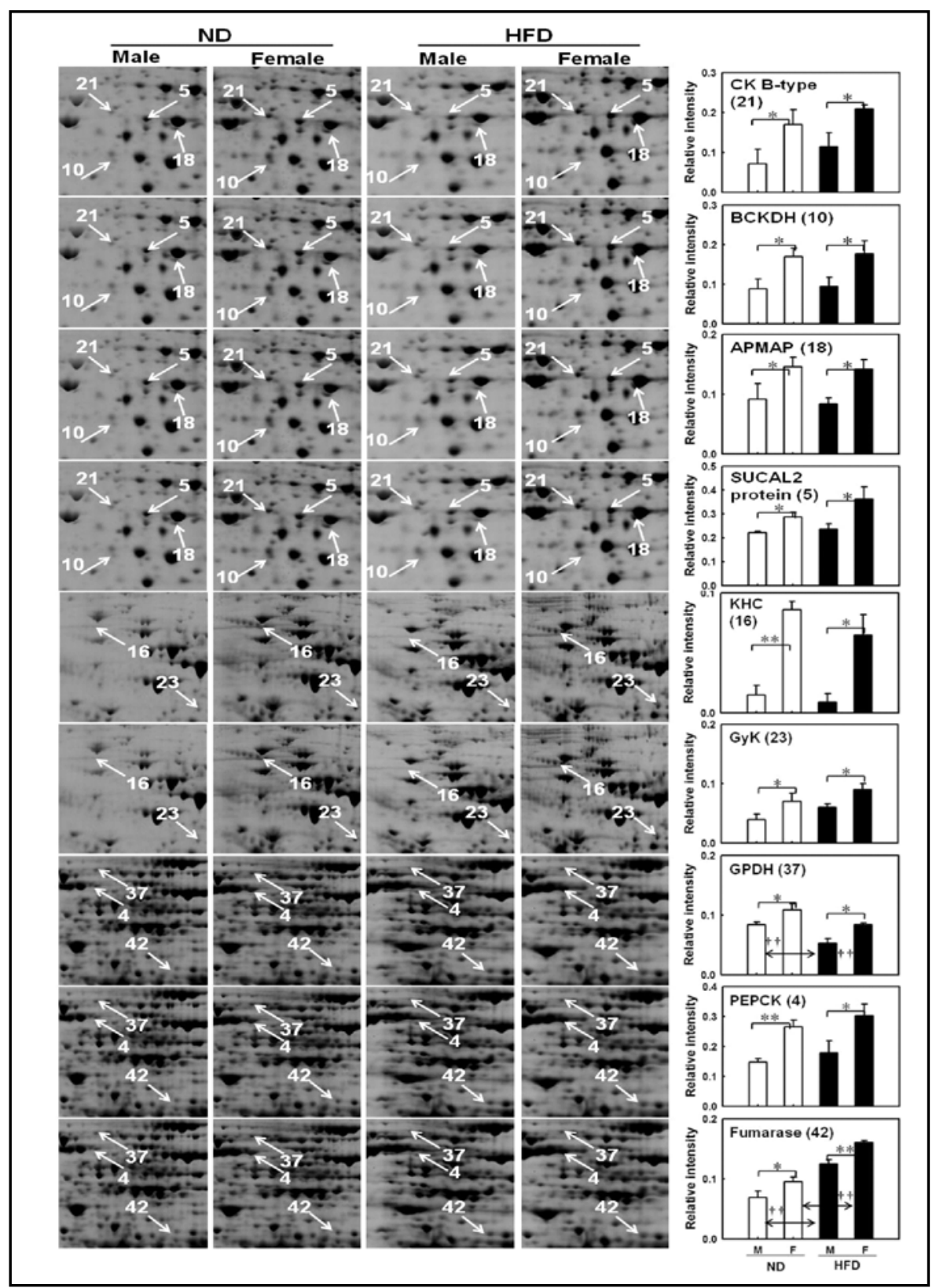

that optimal GLUT4 translocation in response to insulin requires intact actin filaments [51, 52]. Semiz et al. (2003) [53] found that kinesin mediates insulin-stimulated GLUT4 movement on adipocyte cell microtubules. Recent data have also suggested a connection between disruption of intraflagellar transport and obesity, as evidenced by the discovery of proteins associated with human obesity [54]. Therefore, due to striking differences in protein levels of kinesin between genders, this protein can be used as a potential anorectic marker for determination of gender difference in BAT, irrespective of dietary conditions.

We also found higher protein levels of creatine kinase (CK) in females (Fig. 4). In cold adaptation in BAT, upregulation of ATP synthase can be a response of the tissue to sustain the ATP availability needed for housekeeping

Choi/Oh/Choi/Mukherjee/Wang/Liu/Yun 
Fig. 5. Differentially expressed BAT proteins showing gender difference in both ND and HFD rats with opposite regulation patterns. Data are exhibited as mean values \pm SD of volume density (\%) of the changed spot in 3 individual gels using pooled BAT from 7 rats per group. Statistical significance between male $(\mathrm{M})$ and female (F) rats was determined by a $t$-test, where $p$ values were ${ }^{*} p<0.05$ and ${ }^{* *} p<0.01$ and significance between ND and HFD rats is represented by ${ }^{\dagger} p<0.05$ and ${ }^{\dagger} p<0.01$. For abbreviation of each protein name, see Table 3. Arabic numerals in parenthesis of bar graphs indicate spot numbers in zoonin gel images.

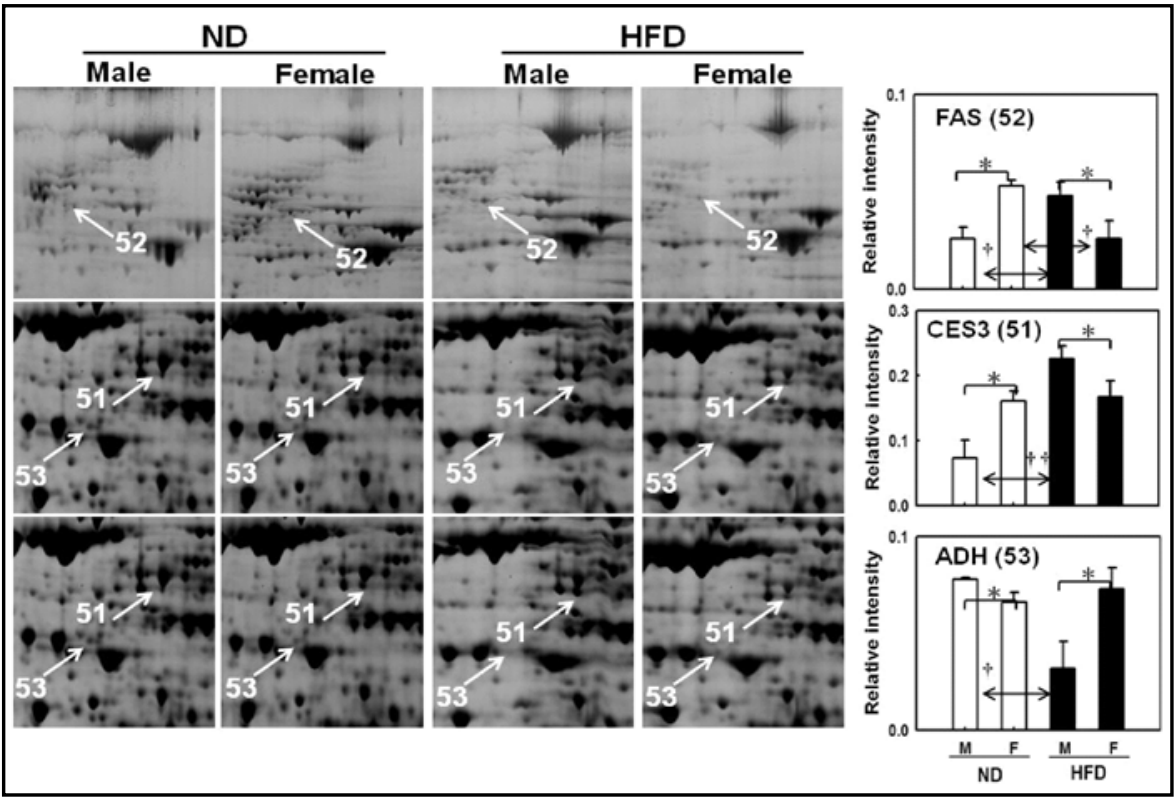

Fig. 6. Proteins showing a genderdifference in the regulation patterns in only ND or HFD rats, and proteins showing no gender-difference, but HFD response. Data are exhibited as mean values \pm SD of volume density $(\%)$ of the changed spot in 3 individual gels using pooled BAT from 7 rats per group. Statistical significance between male $(\mathrm{M})$ and female $(\mathrm{F})$ rats was determined by a $t$-test, where $p$ values were ${ }^{*} p<0.05$ and ${ }^{* *} p<0.01$ and significant differences between ND and HFD rats are represented by ${ }^{\dagger} p<0.05$ and ${ }^{\dagger} p<0.01$. For abbreviation of each protein name, see Table 3. Arabic numerals in parenthesis of bar graphs indicate spot numbers in zoon-in gel images.

Fig. 7. Proteins that were HFD-responsive, but not expressed in a gender-dependent manner. Data are exhibited as mean values \pm SD of volume density (\%) of the changed spot in 3 individual gels using pooled BAT from 7 rats per group. Statistical significance between ND and HFD rats is represented by ${ }^{\dagger} p<0.05$ and ${ }^{\dagger} p<0.01$. For abbreviation of each protein name, see Table 3. Arabic numerals in parenthesis of bar graphs indicate spot numbers in zoon-in gel images.

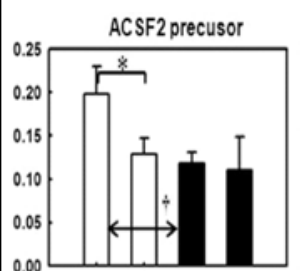

ICDH3 gamma

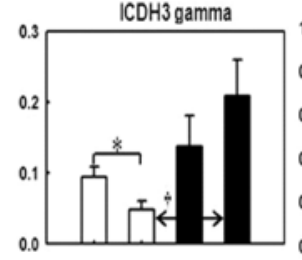

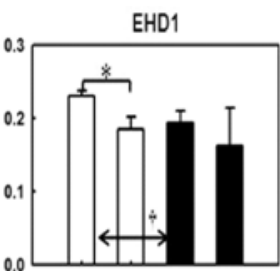

DLD precursor

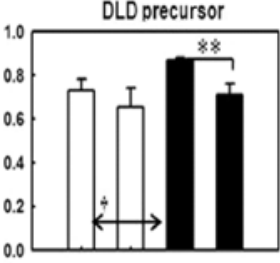

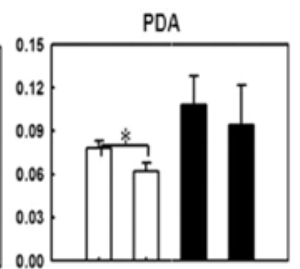

ACLYPROTEIN

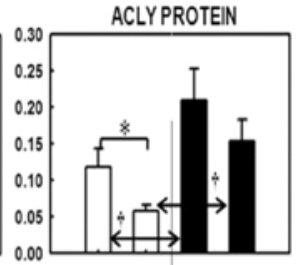

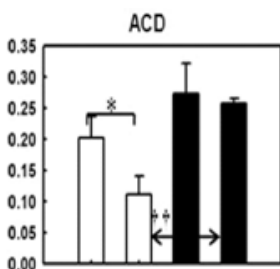

AK2

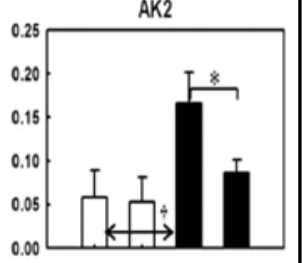

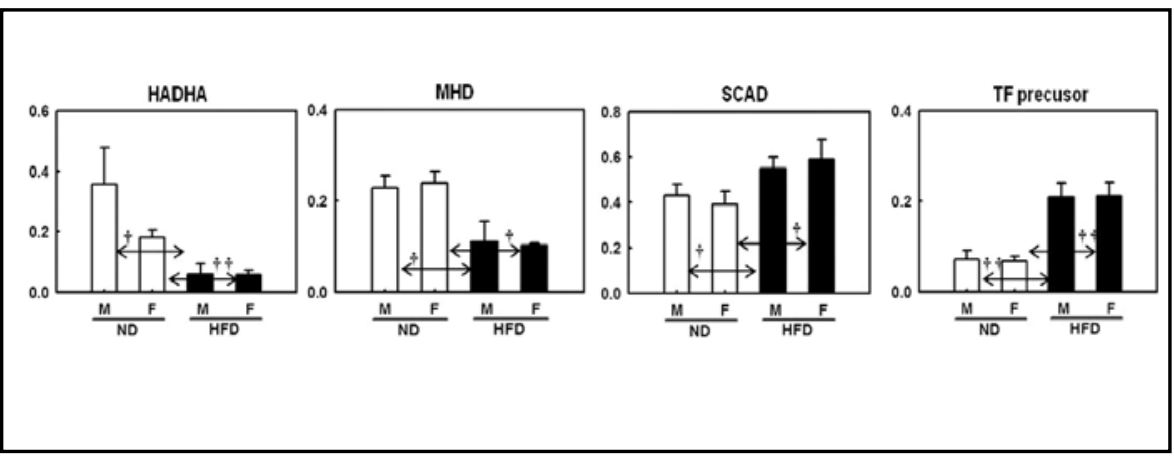

of cold-adapted BAT. Up-regulation of ATP synthase together with down-regulation of $\mathrm{CK}$ is an event that occurs in BAT. The up-regulation of ATP synthase points out the competition that exists between 2 proton electrochemical gradient-consuming systems, including one that is energy-conserving (ATP synthase), while the

other one dissipates energy (UCP1). Higher levels of CK in female BAT suggest a greater capacity for use of phosphocreatine as an energy buffer providing ATP for cytosolic reactions during uncoupling reactions in HFDinduced obese rats.

The higher levels of enzymes involved in the glycerol 


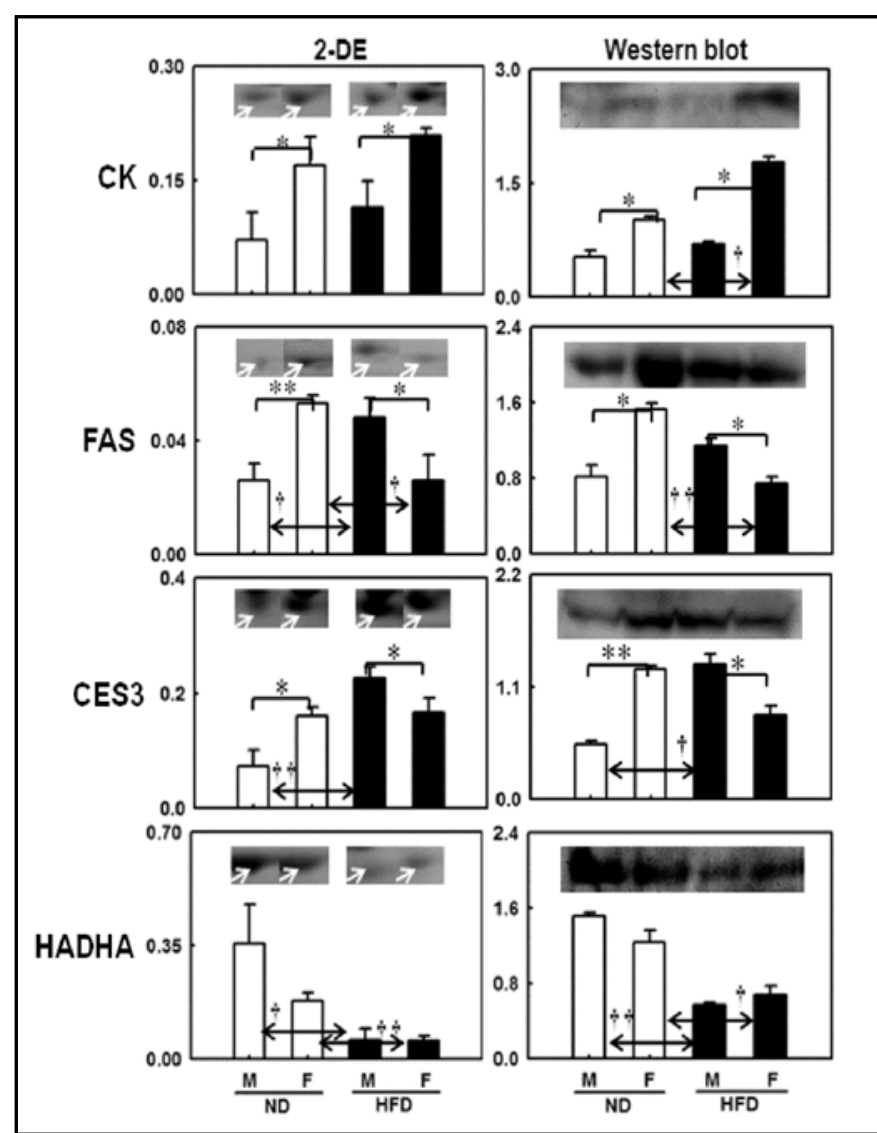

Fig. 8. Validation of differentially regulated BAT proteins in male $(\mathrm{M})$ and female $(\mathrm{F})$ rats in response to HFD by immunoblot analysis. Levels of 4 proteins identified from 2-DE analysis were established using pooled BAT samples from 7 rats per group. Data are representative of 3 independent experiments. For abbreviation of each protein name, see abbreviations.

pathway are of significant interest. Activation of BAT thermogenesis requires hydrolysis of endogenous triacylglycerols for production of fatty acids (FA), which are both substrates and uncoupling messengers for BAT mitochondria [55]. Therefore, maintenance of adequate stores of triacylglycerols, through esterification of newly synthesized or preformed FA via glycerol-3-phosphate (G3P), appears to be essential for normal functioning of BAT. One of the sources of G3P for acylation is the glycerol produced by hydrolysis of stored triacylglycerols or taken up by tissue from the circulation that is directly phosphorylated to G3P by glycerolkinase. Stimulation of glycerolkinase $(\mathrm{GyK})$ gene expression at the pretranslational level, with increased enzyme activity, mediated by $\beta$-adrenoreceptors under conditions of a sustained increase in BAT sympathetic flow has been demonstrated [55]. Our finding of higher protein levels of GyK together with phosphoenolpyruvate carboxylkinase (PEPCK) (Fig. 4), which is a regulatory enzyme of glyceroneogenesis, supports this result. We

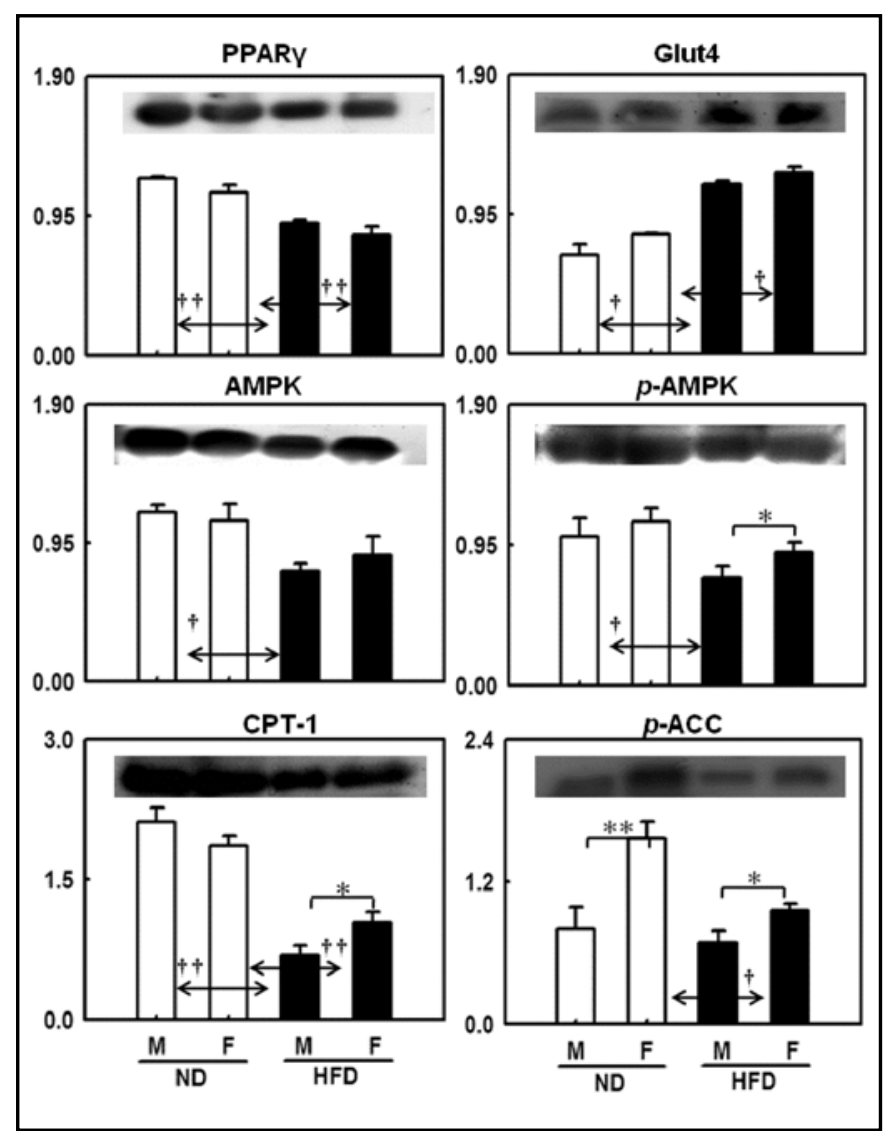

Fig. 9. Differentially regulated BAT proteins of metabolic importance in male $(\mathrm{M})$ and female $(\mathrm{F})$ rats in response to HFD by immunoblot analysis. Levels of 6 important BAT proteins were established using pooled BAT samples from 7 rats per group. Data are representative of 3 independent experiments. For abbreviation of each protein name, see abbreviations.

also found that higher levels of glycerol-3-phosphate dehydrogenase (GPDH) were observed in females (Fig. 4). However, our results strongly argue against the observations that overexpressions of GPDH, GyK or PEPCK led to diet-induced insulin resistance and obesity in white adipose or liver tissue (Fig. 4) [56, 57]. Taken together, the role of these enzymes in BAT in determination of the fate of glycerol (maintenance of FFA and consequent uncoupling messengers for BAT) is likely to differ from that in other tissues (TG accumulation using glycerol as a substrate). Therefore, results of genderdifferent expression of GyK or PEPCK suggest a greater thermogenic activity in female rats.

One of the most striking results in our proteomic study is the finding of different regulation patterns of 2 lipogenic proteins. Protein levels of malic enzyme (ME) and fatty acid synthase (FAS) in HFD-fed females were strikingly lower than in males (Fig. 3 and Fig. 5). Lower ME levels found in the present study may suggest relatively less fat accumulation in both ND- and HFD-

Choi/Oh/Choi/Mukherjee/Wang/Liu/Yun 


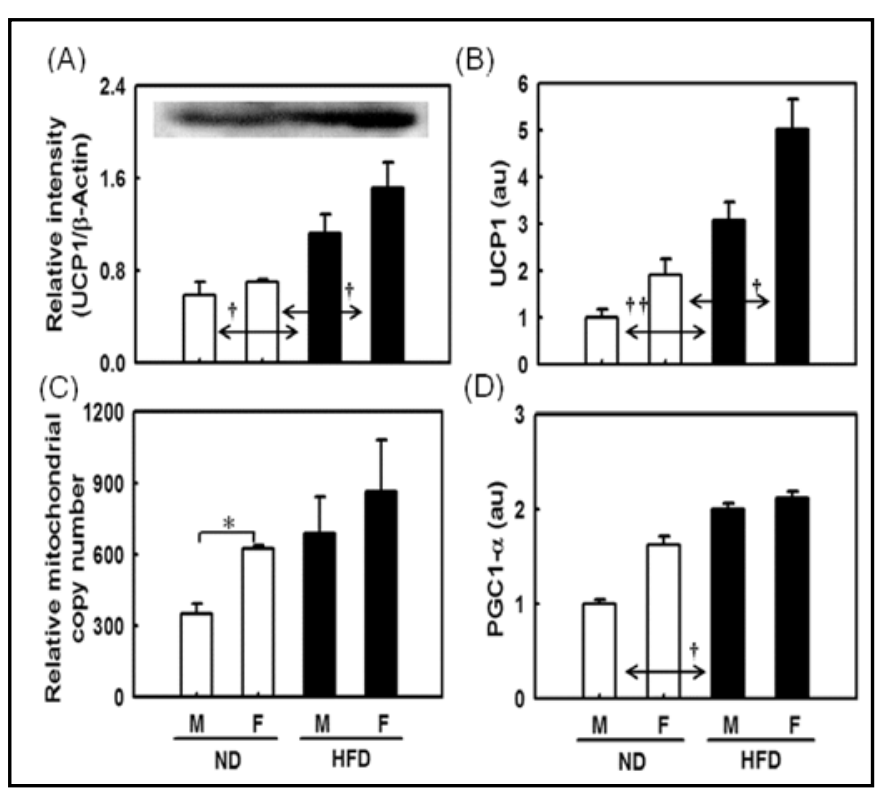

Fig. 10. Comparison of protein (A) and mRNA levels of UCP1 (B), as well as PGC1- $\alpha$ (C) and mitochondrial content (D) in male (M) and female (F) rats in response to HFD. Protein levels were determined by Western blot analysis and mRNA levels were measured using real-time PCR. All were established using pooled BAT samples from 7 rats per group. Data are representative of 3 independent experiments. For abbreviation of each protein name, see abbreviations.

fed female rats [58]. Protein levels of FAS in BAT of male rats were higher in HFD rats than in ND rats, but not in BAT of female rats. Plasma lipid profiles were very similar between the genders (Table 2); therefore, it is likely that the excess lipid deposition in BAT of male rats may not be due to uptake of triacylglycerol from plasma, but due to higher lipogenesis mediated by increased FAS levels within the tissue. In view of the apparent relationship between thermogenesis and lipogenesis in BAT, it might be expected that rates of fatty acid synthesis in BAT of females would be lower in obese rats than in males. It was consequently suggested that BAT might make a major contribution to development of obesity in male rats by virtue of the export of fatty acids for storage in WAT.

Compared with males, down-regulation of aldose reductase (AR) in females is likely to contribute favorably to carbohydrate metabolism (Fig. 3). In response to the chronic hyperglycemia found in diabetics, glucose flux through the polyol pathway is significantly increased. Up to $33 \%$ of total glucose utilization in some tissues can occur through the polyol pathway. Glucose concentrations are often elevated in diabetics and AR has long been believed responsible for diabetic complications involving a number of organs.
We also found lower levels of 2 proteins involved in the mitochondrial respiratory chain, electron-transfer flavoprotein (ETF), and NADH dehydrogenase (ubiquinone) flavoprotein 1 (NDUFV1) in females (Fig. 3 ). Although defects in these proteins are a common cause of mitochondrial dysfunction, down-regulation of these proteins in females is likely to influence enhanced uncoupling capacity in females.

We also detected higher levels of 3 different enzymes of the TCA cycle (fumarase, isocitrate dehydrogenase 3 , and Sucla2) in female rats when fed a HFD (Fig. 4 and Fig. 6). This was likely to increase in $\beta$-oxidation enzymes in females, suggesting more complete oxidation of fatty acids. Without an increase in TCA cycle enzymes, higher levels of $\beta$-oxidation enzymes would result in incomplete fat oxidation, thereby impairing mitochondrial functions. Up-regulation of branched-chain $\alpha$-keto acid dehydrogenase complex (BCKADC), which is a combination of enzymes responsible for degradation of branched chain amino acids, is also a similar result, because this complex is known to be analogous to the alpha-ketoglutarate dehydrogenase complex in the TCA cycle (Fig. 4).

Of particular interest, higher levels were detected in adipocyte plasma membrane-associated protein (APMAP) in female BAT in both ND and HFD rats (Fig. 4). Although the precise biological function of APMAP is unknown at present, it is considered a marker of adipocyte differentiation that is mostly present in mature adipocytes [59]. However, this was found only in WAT, and not in BAT. Together with the observation that female rats had higher BAT content than males, another clue for higher thermogenic activity in females could be derived from differential expression of APMAP between genders when both were exposed to HFD.

The gender-dependent protein levels of GRP75 are another interesting finding in this study. GRP75 is a heatshock cognate protein, which is primarily localized to the mitochondria but also exists in the endoplasmic reticulum (ER), plasma membrane, and cytoplasmic vesicles. This protein plays a role in cell proliferation, stress response, and maintenance of mitochondria [60]. A protective mechanism used by cells in adaptation to stress of the ER is induction of members of the GRP family. Induction of mammalian GRP proteins in response to ER stress involves a complex network of regulators and novel mechanisms. Elucidation of GRP function and regulation opens up new therapeutic approaches to diseases associated with ER stress and cancer [61]. Pathological conditions, such as tumor growth, have been reported to 
show correlation with GRP over-expression [61, 62] Therefore, our findings raise the possibility that higher protein levels of GRP75 in males may reflect a more severe state of obesity (Fig. 3).

Proteins showing gender-difference with opposite regulation patterns in $N D$ and HFD rats

HFD-responsive up-regulation of carboxylesterases 3 (CES3) only in males is also an interesting result in this study (Fig. 5). CESs constitute an enzyme family that converts a carboxylic ester to an alcohol and a carboxylate, and have recently been suggested to play a role in lipolysis. Jernas and coworkers [63] recently demonstrated that expression of the CES1 gene is highly regulated in human adipose tissue, with increased levels in obese subjects, and decreased levels during weight loss. However, differential expression patterns of these enzymes in mammalian BAT have not been demonstrated. Our proteomic analysis of rat BAT pointed indicated that HFD-fed rats showed higher CES3 expression than in ND-fed mice only in males, reflecting a sign of higher lipid accumulation in HFD-fed male rats, but not in females.

Opposing regulation responses of aldehyde dehydrogenase (ADH) to HFD between genders is worthy of discussion (Fig. 5). In males, levels of ADH were markedly reduced in HFD rats, whereas no significant changes were observed in females. One possible explanation for this result could be the greater detoxifying role in lipid-derived oxidative stress because $\mathrm{ADH}$ was identified as a key component of the detoxification pathway of aldehydes arising from lipid peroxidation events [64].

Results of immunoblot analysis clearly supported the proteomic results, in that identical expression patterns for 4 selected proteins were observed (e.g. CK, CES3, FAS, and HADHA). To confirm higher thermogenic activity in female BAT obtained from the proteomic study, we compared the mitochondrial content, levels of UCP1 (marker for energy expenditure), and PGC1- $\alpha$ (marker for mitochondrial biogenesis and estrogen receptor activator) between genders. Somewhat to our surprise, we found that expression of all of these factors showed gender-difference. In particular, female rats exhibited significantly greater stimulation of UCP1 expression in both protein and mRNA levels than did males (Fig. 10).

In this study, we found interesting changes in the levels of estrogen and testosterone in response to HFD feeding. Levels of estrogen in both males and females were increased in HFD-fed rats. A higher magnitude of increase in females $(30 \%)$ than in males $(15 \%)$, compared with ND rats, was observed (Table 2). In contrast, levels of testosterone in males showed a striking decrease (53\%), but showed a detectable increase in females (13\%). Taken together, maintenance of high levels of estrogen also supports increased energy expenditure in HFD-fed female rats. Our finding is in agreement with earlier results showing that mice with deficient estrogen receptors or aromatase accumulated higher levels of fat than their littermates $[65,66]$.

In conclusion, Current proteomic analysis of BAT in HFD-induced obesity allowed us to identify 48 proteins showing gender-specific regulation in male and female rats. Although some of these proteins have already been linked to obesity, gender-dependent regulation patterns of most identified proteins are reported for the first time in this study. We found conclusive results showing greater expression of numerous proteins directly or indirectly involved in thermogenic action in females. In addition, traits of lower fat synthesis and higher oxidation activity in females were suggested, although the activity of each enzyme activity was not been determined in this study. We believe that gender-different protein regulation in BAT resulted, at least in part, from sex hormones. For example, estrogen might down regulate lipogenic enzymes and shift partition of FFA toward oxidation and away from TG storage by activation of AMPK. In conclusion, the present proteomic research into gender-dimorphic pathophysiological mechanisms will aid in improvement of gender awareness in the health care system and in implementation of evidence-based gender specific clinical recommendations.

\section{Abbreviations}

ACC (Acetyl-CoA carboxylase); AMPK (AMPactivated protein kinase); BAT (brown adipose tissue); 2-DE (two-dimensional electrophoresis); CPT (carnitine palmitoyltransferase); GLUT (glucose transporter); HFD (high fat diet); ND (normal diet); PGC (PPAR gamma coactivator); PPAR (peroxisome proliferator-activator receptor); UCP (uncoupling protein).

\section{Acknowledgements}

This research was supported by the Mid-career Researcher Program (grant number 2011-0000509) and SRC program (Center for Food \& Nutritional Genomics:

Choi/Oh/Choi/Mukherjee/Wang/Liu/Yun 
grant number 2011-0000914) of the National Research Foundation of Korea (NRF) funded by the Ministry of Education, Science and Technology. Duk Kwon Choi, Tae
Seok Oh, Jung-Won Choi, Rajib Mukherjee, Xia Wang, and Hao Liu received the BK21 scholarship.

\section{References}

1 Kautzky-Willer A, Handisurya A Metabolic diseases and associated complications: Sex and gender matter! Eur J Clin Invest 2009;39:631-648.

- 2 Sweeting H, West P, Young R: Obesity among scottish 15 year olds 1987-2006: Prevalence and associations with socioeconomic status, well-being and worries about weight. BMC Public Health 2008;8:404-410.

-3 Blouin K, Boivin A, Tchernof A: Androgens and body fat distribution. $\mathrm{J}$ Steroid Biochem Mol Biol 2008;108:272280.

-4 Nguyen TT, Hernandez Mijares A, Johnson CM, Jensen MD: Postprandial leg and splanchnic fatty acid metabolism in nonobese men and women. Am J Physiol 1996;271:E965-972.

-5 Blaak E: Gender differences in fat metabolism. Curr Opin Clin Nutr Metab Care 2001;4:499-502.

-6 Llado I, Estrany ME, Rodriguez E, Amengual B, Roca P, Palou A: Effects of cafeteria diet feeding on beta3adrenoceptor expression and lipolytic activity in white adipose tissue of male and female rats. Int $\mathrm{J}$ Obes Relat Metab Disord 2000;24:1396-1404.

-7 Llado I, Rodriguez-Cuenca S, Pujol E, Monjo M, Estrany ME, Roca P, Palou A: Gender effects on adrenergic receptor expression and lipolysis in white adipose tissue of rats. Obes Res 2002;10:296-305.

-8 Shi H, Seeley RJ, Clegg DJ: Sexual differences in the control of energy homeostasis. Front Neuroendocrinol 2009;30:396-404.

-9 Zillikens MC, Yazdanpanah M, Pardo LM, Rivadeneira F, Aulchenko YS, Oostra BA, Uitterlinden AG, Pols HA, van Duijn CM: Sex-specific genetic effects influence variation in body composition. Diabetologia 2008;51:2233-2241.

10 Schousboe K, Willemsen G, Kyvik KO, Mortensen J, Boomsma DI, Cornes BK, Davis CJ, Fagnani C, Hjelmborg J, Kaprio J, De Lange M, Luciano M, Martin NG, Pedersen N, Pietilainen KH, Rissanen A, Saarni S, Sorensen TI, Van Baal GC, Harris JR: Sex differences in heritability of bmi: A comparative study of results from twin studies in eight countries. Twin Res 2003;6:409-421.
Arciero PJ, Goran MI, Poehlman ET: Resting metabolic rate is lower in women than in men. J Appl Physiol 1993;75:2514-2520.

12 Tarnopolsky MA, Atkinson SA, Phillips SM, MacDougall JD: Carbohydrate loading and metabolism during exercise in men and women. J Appl Physiol 1995;78:1360-1368.

Komi PV, Karlsson J: Skeletal muscle fibre types, enzyme activities and physical performance in young males and females. Acta Physiol Scand 1978;103:210-218.

$\checkmark 14$ Henderson GC, Fattor JA, Horning MA, Faghihnia N, Johnson ML, Luke-Zeitoun $\mathrm{M}$, Brooks GA: Glucoregulation is more precise in women than in men during postexercise recovery. Am J Clin Nutr 2008;87:1686-1694.

15 Rodriguez AM, Palou A: Uncoupling proteins: Gender dependence and their relation to body weight control. Int $\mathrm{J}$ Obes Relat Metab Disord 2004;28:500502.

Roca P, Rodriguez AM, Oliver P, Bonet ML, Quevedo S, Pico C, Palou A: Brown adipose tissue response to cafeteria dietfeeding involves induction of the ucp2 gene and is impaired in female rats as compared to males. Pflugers Arch 1999;438:628-634.

Bjorntorp P: Hormonal control of regional fat distribution. Hum Reprod 1997;12 Suppl 1:21-25.

Rodriguez-Cuenca S, Pujol E, Justo R, Frontera M, Oliver J, Gianotti M, Roca P: Sex-dependent thermogenesis, differences in mitochondrial morphology and function, and adrenergic response in brown adipose tissue. J Biol Chem 2002;277:42958-42963.

19 Allan CA, Strauss BJ, Burger HG, Forbes EA, McLachlan RI: Testosterone therapy prevents gain in visceral adipose tissue and loss of skeletal muscle in nonobese aging men. J Clin Endocrinol Metab 2008;93:139-146.

20 Quevedo S, Roca P, Pico C, Palou A: Sexassociated differences in cold-induced ucp1 synthesis in rodent brown adipose tissue. Pflugers Arch 1998;436:689-695. Rodriguez AM, Quevedo-Coli S, Roca P, Palou A: Sex-dependent dietary obesity, induction of ucps, and leptin expression in rat adipose tissues. Obes Res 2001;9:579-588.
22 Valle A, Catala-Niell A, Colom B, GarciaPalmer FJ, Oliver J, Roca P: Sex-related differences in energy balance in response to caloric restriction. Am J Physiol Endocrinol Metab 2005;289:E15-22.

23 Macotela Y, Boucher J, Tran TT, Kahn CR: Sex and depot differences in adipocyte insulin sensitivity and glucose metabolism. Diabetes 2009;58:803-812. Wang GJ, Volkow ND, Telang F, Jayne M, Ma Y, Pradhan K, Zhu W, Wong CT, Thanos PK, Geliebter A, Biegon A, Fowler JS: Evidence of gender differences in the ability to inhibit brain activation elicited by food stimulation. Proc Natl Acad Sci U S A 2009;106:1249-1254.

-25 Matsuda Y, Tanioka T, Yoshioka T, Nagano T, Hiroi T, Yoshikawa K, Okabe K, Nagamine I, Takasaka Y: Gender differences in association of plasma adiponectin with obesity reflect resultant insulin resistance in non-diabetic japanese patients with schizophrenia. Psychiatry Clin Neurosci 2005;59:266-273.

-26 Fujiki N, Yoshida Y, Zhang S, Sakurai T, Yanagisawa M, Nishino S: Sex difference in body weight gain and leptin signaling in hypocretin/orexin deficient mouse models. Peptides 2006;27:2326-2331.

27 Havel PJ, Kasim-Karakas S, Dubuc GR, Mueller W, Phinney SD: Gender differences in plasma leptin concentrations. Nat Med 1996;2:949950 .

-28 Clegg DJ, Riedy CA, Smith KA, Benoit SC, Woods SC: Differential sensitivity to central leptin and insulin in male and female rats. Diabetes 2003;52:682-687.

$>29$ Frank S, Laharnar N, Kullmann S, Veit R, Canova C, Hegner YL, Fritsche A, Preissl H: Processing of food pictures: Influence of hunger, gender and calorie content. Brain Res 2010;1350:159-166. Magkos F, Mittendorfer B: Gender differences in lipid metabolism and the effect of obesity. Obstet Gynecol Clin North Am 2009;36:245-265, vii.

-31 Hong J, Stubbins RE, Smith RR, Harvey AE, Nunez NP: Differential susceptibility to obesity between male, female and ovariectomized female mice. Nutr J 2009;8:11.

32 Legato MJ: Gender-specific aspects of obesity. Int J Fertil Womens Med 1997;42:184-197. 
33 Gayle DA, Desai M, Casillas E, Beloosesky R, Ross MG: Gender-specific orexigenic and anorexigenic mechanisms in rats. Life Sci 2006;79:1531-1536.

$\$ 34$ Catala-Niell A, Estrany ME, Proenza AM, Gianotti M, Llado I: Skeletal muscle and liver oxidative metabolism in response to a voluntary isocaloric intake of a high fat diet in male and female rats. Cell Physiol Biochem 2008;22:327-336.

$\$ 35$ Justo R, Boada J, Frontera M, Oliver J, Bermudez J, Gianotti M: Gender dimorphism in rat liver mitochondrial oxidative metabolism and biogenesis. Am J Physiol Cell Physiol 2005;289:C372378

\36 Valle A, Guevara R, Garcia-Palmer FJ, Roca P, Oliver J: Sexual dimorphism in liver mitochondrial oxidative capacity is conserved under caloric restriction conditions. Am J Physiol Cell Physiol 2007;293:C1302-1308.

$\checkmark 37$ Miike K, Aoki M, Yamashita R, Takegawa Y, Saya H, Miike T, Yamamura $\mathrm{K}$ : Proteome profiling reveals gender differences in the composition of human serum. Proteomics 2010;10:2678-2691.

$\$ 38$ Eidelman O, Jozwik C, Huang W, Srivastava M, Rothwell SW, Jacobowitz DM, Ji X, Zhang X, Guggino W, Wright J, Kiefer J, Olsen C, Adimi N, Mueller GP, Pollard HB: Gender dependence for a subset of the low-abundance signaling proteome in human platelets. Hum Genomics Proteomics 2010;2010:164906.

39 Metskas LA, Kulp M, Scordilis SP: Gender dimorphism in the exercise-naive murine skeletal muscle proteome. Cell Mol Biol Lett 2010;15:507-516.

40 Amelina H, Cristobal S: Proteomic study on gender differences in aging kidney of mice. Proteome Sci 2009;7:16.

$>4$ Martins-de-Souza D, Schmitt A, Roder R, Lebar M, Schneider-Axmann T, Falkai $\mathrm{P}$, Turck CW: Sex-specific proteome differences in the anterior cingulate cortex of schizophrenia. J Psychiatr Res 2010;44:989-991.

42 Shetty J, Naaby-Hansen S, Shibahara H, Bronson R, Flickinger CJ, Herr JC: Human sperm proteome: Immunodominant sperm surface antigens identified with sera from infertile men and women. Biol Reprod 1999;61:61-69.

$>43$ Schmid GM, Converset V, Walter N, Sennitt MV, Leung KY, Byers H, Ward M, Hochstrasser DF, Cawthorne MA, Sanchez JC: Effect of high-fat diet on the expression of proteins in muscle, adipose tissues, and liver of $\mathrm{c} 57 \mathrm{bl} / 6$ mice. Proteomics 2004;4:2270-2282.
Oh Tim DH, Choi DK, Wang $X$, Choi JW, Yun JW: Differential expression of adipose tissue proteins between obesity-susceptible and -resistant rats fed a high-fat diet. Proteomics 2011;11:1429-1448.

$\$ 45$ Bradford MM: A rapid and sensitive method for the quantitation of microgram quantities of protein utilizing the principle of protein-dye binding. Anal Biochem 1976;72:248-254.

46

Kim DH, Choi JW, Joo JI, Wang X, Choi DK, Oh TS, Yun JW: Changes in expression of skeletal muscle proteins between obesity-prone and obesityresistant rats induced by a high-fat diet. J Proteome Res 2011;10:1281-1292.

Borras C, Sastre J, Garcia-Sala D, Lloret A, Pallardo FV, Vina J: Mitochondria from females exhibit higher antioxidant gene expression and lower oxidative damage than males. Free Radic Biol Med 2003;34:546-552.

$\$ 48$ Vale RD, Reese TS, Sheetz MP: Identification of a novel force-generating protein, kinesin, involved in microtubulebased motility. Cell 1985;42:39-50.

49 Kull FJ, Sablin EP, Lau R, Fletterick RJ, Vale RD: Crystal structure of the kinesin motor domain reveals a structural similarity to myosin. Nature 1996;380:550-555.

-50 Skowronek KJ, Kocik E, Kasprzak AA: Subunits interactions in kinesin motors. Eur J Cell Biol 2007;86:559-568.

Wang Q, Bilan PJ, Tsakiridis T, Hinek A, Klip A: Actin filaments participate in the relocalization of phosphatidylinositol3kinase to glucose transporter-containing compartments and in the stimulation of glucose uptake in 3t3-11 adipocytes. Biochem J 1998;331:917-928.

$\checkmark 52$ Omata W, Shibata H, Li L, Takata K, Kojima I: Actin filaments play a critical role in insulin-induced exocytotic recruitment but not in endocytosis of glut4 in isolated rat adipocytes. Biochem J 2000;346:321-328.

53 Semiz S, Park JG, Nicoloro SM, Furcinitti P, Zhang C, Chawla A, Leszyk J, Czech MP: Conventional kinesin kif5b mediates insulin-stimulated glut4 movements on microtubules. EMBO J 2003;22:23872399.

\$5 Davenport JR, Watts AJ, Roper VC, Croyle MJ, van Groen T, Wyss JM, Nagy TR, Kesterson RA, Yoder BK: Disruption of intraflagellar transport in adult mice leads to obesity and slow-onset cystic kidney disease. Curr Biol 2007;17:15861594.

55 Festuccia WT, Guerra-Sa R, Kawashita NH, Garofalo MA, Evangelista EA, Rodrigues V, Kettelhut IC, Migliorini RH: Expression of glycerokinase in brown adipose tissue is stimulated by the sympathetic nervous system. Am J Physiol Regul Integr Comp Physiol 2003;284:R1536-1541.
Franckhauser S, Munoz S, Elias I, Ferre T, Bosch F: Adipose overexpression of phosphoenolpyruvate carboxykinase leads to high susceptibility to diet-induced insulin resistance and obesity. Diabetes 2006;55:273-280.

MacDougald OA, Burant CF: Obesity and metabolic perturbations after loss of aquaporin 7 , the adipose glycerol transporter. Proc Natl Acad Sci U S A 2005;102:10759-10760.

$\checkmark 58$ Smith RG, Gauthier DA, Dennis DT, Turpin DH: Malate- and pyruvatedependent fatty acid synthesis in leucoplasts from developing castor endosperm. Plant Physiol 1992;98:12331238 .

Albrektsen T, Richter HE, Clausen JT, Fleckner J: Identification of a novel integral plasma membrane protein induced during adipocyte differentiation. Biochem J 2001;359:393-402.

60 Ran Q, Wadhwa R, Kawai R, Kaul SC, Sifers RN, Bick RJ, Smith JR, PereiraSmith OM: Extramitochondrial localization of mortalin/mthsp70/pbp74/ grp75. Biochem Biophys Res Commun 2000;275:174-179.

61 Lee AS: The glucose-regulated proteins: Stress induction and clinical applications. Trends Biochem Sci 2001;26:504-510.

2 Little E, Ramakrishnan M, Roy B, Gazit G, Lee AS: The glucose-regulated proteins (grp78 and grp94): Functions, gene regulation, and applications. Crit Rev Eukaryot Gene Expr 1994;4:1-18.

Jernas M, Olsson B, Arner P, Jacobson P, Sjostrom L, Walley A, Froguel P, McTernan PG, Hoffstedt J, Carlsson LM: Regulation of carboxylesterase 1 (ces1) in human adipose tissue. Biochem Biophys Res Commun 2009;383:63-67. Demozay D, Rocchi S, Mas JC, Grillo S, Pirola L, Chavey C, Van Obberghen E: Fatty aldehyde dehydrogenase: Potential role in oxidative stress protection and regulation of its gene expression by insulin. J Biol Chem 2004;279:62616270.

Heine PA, Taylor JA, Iwamoto GA, Lubahn DB, Cooke PS: Increased adipose tissue in male and female estrogen receptor-alpha knockout mice. Proc Natl Acad Sci U S A 2000;97:1272912734.

Jones ME, Thorburn AW, Britt KL, Hewitt KN, Wreford NG, Proietto J, Oz OK, Leury BJ, Robertson KM, Yao S, Simpson ER: Aromatase-deficient (arko) mice have a phenotype of increased adiposity. Proc Natl Acad Sci U S A 2000;97:12735-12740. 